\title{
Development of patient specific cardiovascular models predicting dynamics in response to orthostatic stress challenges
}

\author{
Johnny T. Ottesen, Vera Novak, Mette S. Olufsen \\ Department of Science, Systems and Models \\ Roskilde University, \\ 4000 Roskilde, Denmark \\ Division of Gerontology \\ Beth Israel Deaconess Medical School and Harvard University \\ Boston, MA 02215
}

Center for Research in Scientific Computing and Department of Mathematics

North Carolina State University, Raleigh, NC 27695

June 26, 2009 


\begin{abstract}
Physiological realistic models of the controlled cardiovascular system are constructed and validated against clinical data. Special attention is paid to the control of blood pressure, cerebral blood flow velocity, and heart rate during postural challenges, including sit-to-stand and head-up tilt. This study describes development of patient specific models, and how sensitivity analysis and nonlinear optimization methods can be used to predict patient specific characteristics when analyzed using experimental data. Finally, we discuss how a given model can be used to understand physiological changes between groups of individuals and how to use modeling to identify biomarkers.
\end{abstract}

\title{
1 Introduction
}

This manuscript summarizes a series of cardiovascular control models predicting baroreflex and cerebral autonomic regulation of blood flow and pressure during orthostatic postural challenges including sitting to standing (STS) and head-up tilt (HUT) $[24,25,26,27,28,29,30,18,19,20,21,22,5,2]$. During these challenges gravity pools blood from the upper to the lower body. As a result venous return is reduced, leading to a decrease in cardiac stroke volume, a decline in upper body arterial blood pressure (see Fig. 1), an increase in lower body arterial pressure, and an immediate decrease of blood flow to the brain (see Fig. 2). These changes are rapidly counteracted by short-term feedback control mechanisms working to reestablish blood flow and pressure. The main short-term feedback mechanisms involved are autonomic regulation (mainly baroreceptor feedback control) and cerebral autoregulation. The barorecepter system controls blood pressure by regulating heart rate (see Fig. 3), vascular tone (resistance and compliance), and cardiac contractility in response to changes in blood pressure, while cerebral autoregulation controls cerebral blood flow by regulating cerebrovascular tone (resistance and compliance). These control mechanisms are complex and interact in ways that are not yet fully understood. Furthermore, it is believed that their functions are compromized by aging and disease. For example, mean blood flow velocity and pressure show similar dynamics for healthy young and hypertensive elderly subjects, while the pulse velocity (the width - the systolic minus the diastolic value for each pulse wave) in response to standing up is increased for healthy young subjects but not for hypertensive elderly subjects (compare Figs. 1 and 2). It is likely that this widening of the pulse wave (or lack thereof) is a response to changes in the feedback control. 

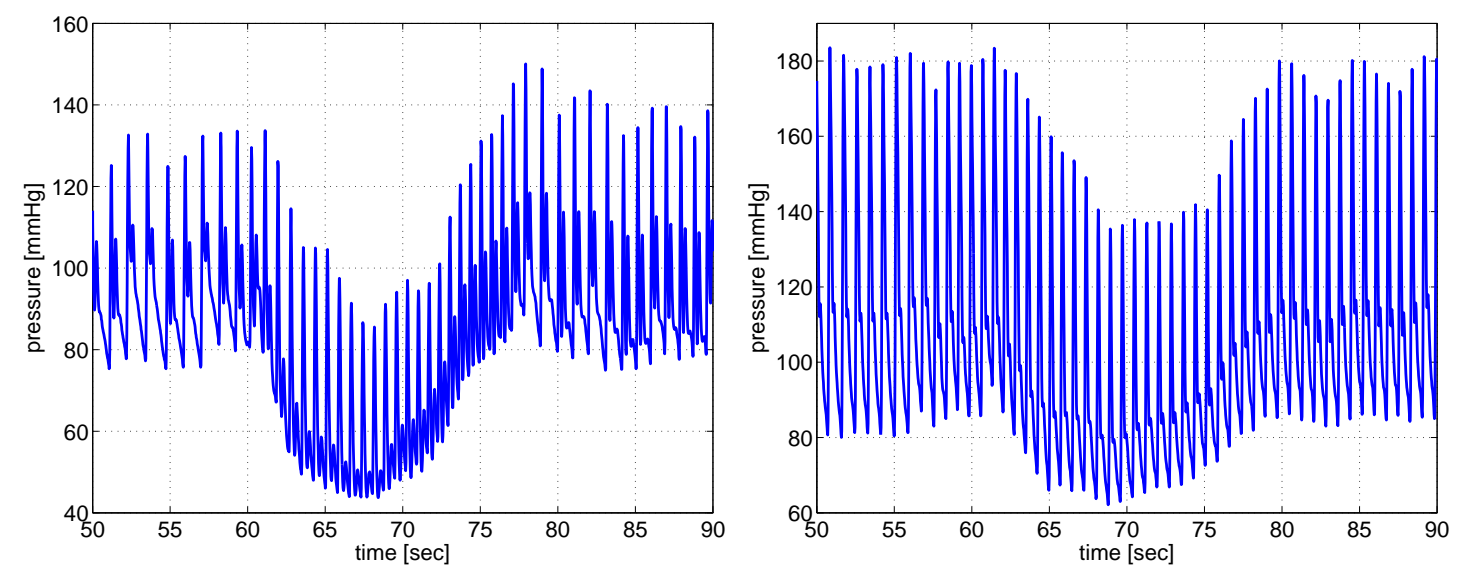

Figure 1: Blood pressure [mmHg] versus time [sec] during STS for a healthy young (left) and a hypertensive elderly (right) subject.
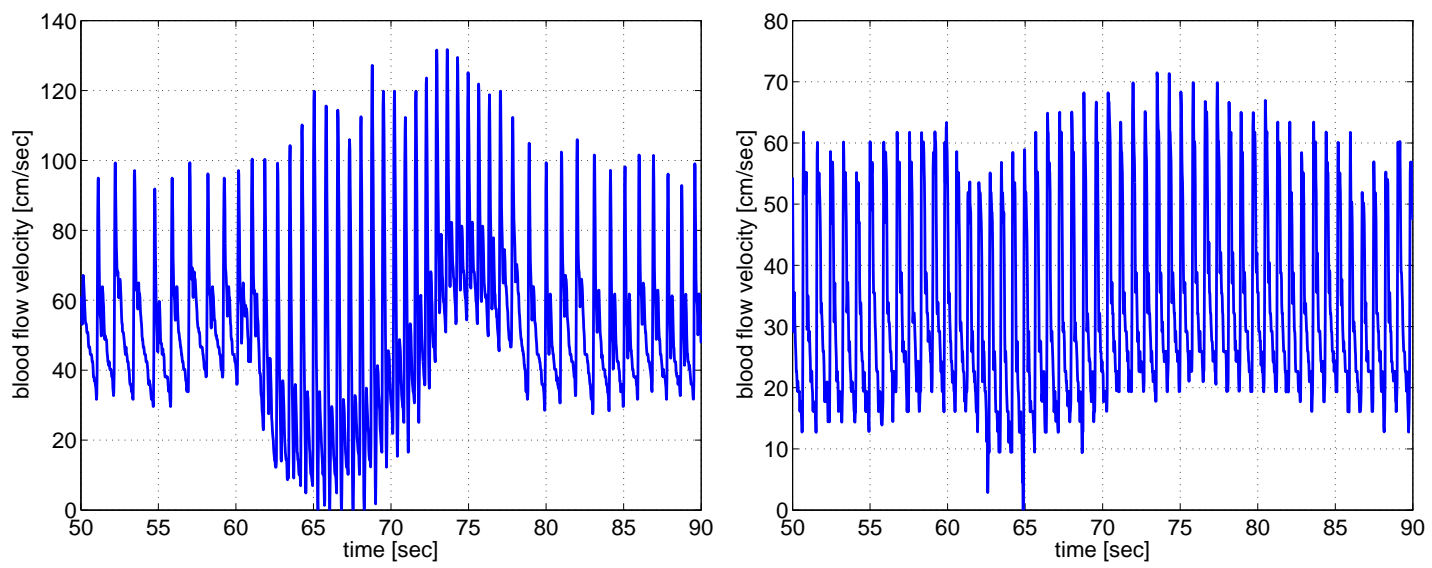

Figure 2: Cerebral blood flow velocity [ $\mathrm{cm} / \mathrm{sec}]$ versus time [sec] during STS for a healthy young (left) and a hypertensive elderly (right) subject. 

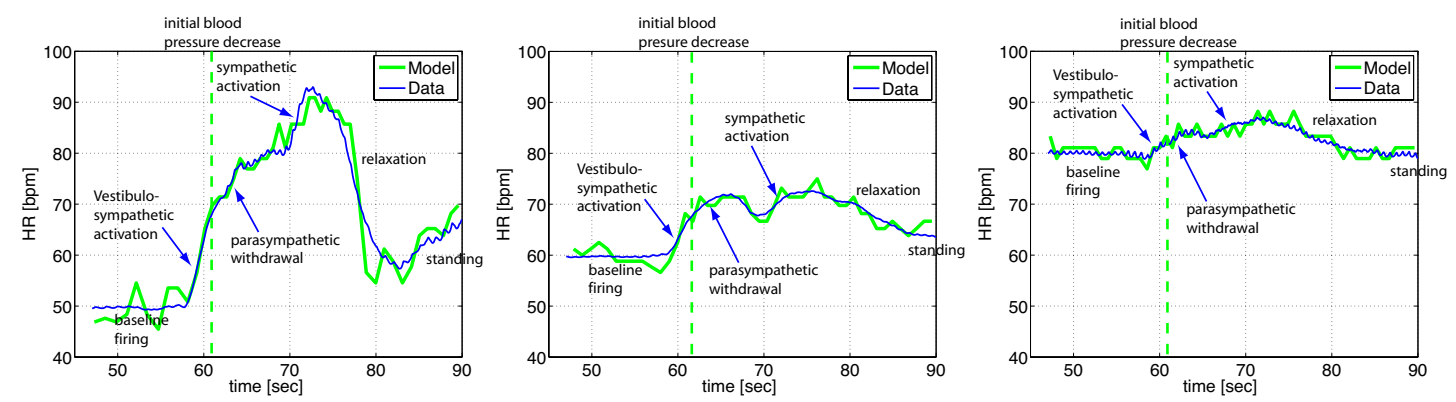

Figure 3: Heart rate [bpm] versus time [sec] for a healthy young (left), a healthy elderly (middle) and a hypertensive elderly (right) subject.

The baroreflex regulation acts through stimulation of baroreceptors located in the carotid and aortic walls. These receptors are stretch receptors, which sense changes in arterial blood pressure. A blood pressure drop leads to decreased firing of the afferent baroreceptor nerves, which gives rise to parasympathetic withdrawal and sympathetic activation. Parasympathetic withdrawal induces a fast increase in heart rate within 1-2 cardiac cycles, while sympathetic stimulation yields a delayed (within 6-8 cardiac cycles) increase in vascular tone (resistance and compliance), cardiac contractility, and a further increase in heart rate $[38,8]$. At the same time cerebrovascular tone is modulated through metabolic vasoregulation (mediated by changes in $\mathrm{CO}_{2}$ ) and myogenic autoregulation (regulation responding to local changes in blood pressure). It is not clear if autonomic control also regulates cerebrovascular tone. Some recent studies $[45,46]$ have indicated that cerebral vascular tone is modulated by stimulation of cholinergic nerves terminating in the cerebral vasculature.

In humans, autonomic and cerebral autoregulation is most often studied by analyzing dynamics during STS and HUT experiments [14, 37]. One difference between these two challenges is that HUT is often carried out using a slow-tilt lasting 5-10 seconds, while STS occurs rapidly over 1-5 seconds. As a result the regulatory response (in particular the parasympathetic portion of the response) to HUT is initiated before the subject is fully tilted thereby preventing excessive drop in blood pressure, while during STS blood is pooled to the legs fast leading to a much larger drop in pressure. This difference in the orthostatic motion impacts the loading of the baroreceptors. During HUT, gravitational forces act between the head and the torso as well as between the torso and the lower body, while STS mainly pools blood to the legs (in elderly a considerable amount of blood may be pooled in the legs during sitting). As a result HUT gives rise to an increased flow 
from the brain to the heart, which for a period of time may increase right atrial pressure stimulating pulmonary baroreceptors, which has potential to lower heart rate. During STS, there is no hydrostatic change between various regions in upper corpus, thus all pooling of blood will be in the legs. Therefore this motion does not give rise to the immediate decrease in heart rate upon standing.

Another difference is that HUT is almost entirely passive, while STS require muscle contraction in the legs activating muscle sympathetic nerves (MSNA), which may increase heart rate before the observed drop in blood pressure. Other explanation for the initial increase in heart rate include stimulation by the vestibular system, and/or by central command.

During HUT and STS experiments typical cardiovascular measurements include: arterial blood pressure, heart rate, and cerebral blood fow velocity measured using Transcranial Doppler ultrasound. These measurements are then used to assess short term autonomic (baroreflex) and cerebral autoregulation. Most common data analysis methods use some form of linear response models. For example, baroreflex sensitivity [36,9] is often assessed using spectral transfer functions relating changes in systolic blood pressure to interbeat intervals. Problems with this method is two-fold; first the method is linear, second it is limited to analysis of the relationships between two signals. Another limitation is that these methods lack the ability to predict how changes in neural responses interact to maintain arterial blood pressure.

In this manuscript we discuss alternative approaches based on nonlinear dynamic mathematical models. Two models will be discussed in detail: an open loop model developed to predict STS and HUT regulation of heart rate and a closed loop model developed to predict blood flow and blood pressure baroreflex and cerebral autoregulation during STS. The heart rate model has been discussed in $[24,25,17,21,22]$ and closed loop model has been discussed in $[30,18,19$, $20,6,31,5]$. Both models were developed to analyze patient specific data, i.e., our goal was to develop models that allow prediction of dynamic quantities including cerebral blood flow velocity, arterial blood pressure, and heart rate. To do so, we estimated a set of nominal parameters for each subject using allometric scaling laws and anthropometric data (height, weight, age gender). Using these nominal values patient specific values were obtained by predicting a subset of parameters minimizing (in a reliable way) the difference between computed and measured values of the observed quantities. The subset of parameters identified using optimization have potential to function as biomarkers, which can reveal significant differences between healthy and diseased subjects (or even within a group of subjects). Not all optimized parameters will be different between groups, but 
those that do have potential to serve as biomarkers characterizing varying states of diseases. Finally, we show dynamics observed during longer time scales $(>35$ $\min$ ) for a subject who experienced syncope. Note, at the onset of syncope blood pressure and heart rate decreases rapidly. We show (see Fig. 15) that our model is able predict a similar behavior for a suitable choice of parameters, i.e., when saturation in the vascular tone is reached.

\section{Methods}

\subsection{Experimental design}

The results reviewed used STS and HUT data from a number of healthy, hypertensive, and elderly subjects. The healthy young and elderly subjects where not treated for any systemic disease and the hypertensive elderly subjects were diagnosed and treated for hypertension but had no history of more than one episode of syncope. Subjects with a history of diabetes, stroke and brain injury, renal liver and other systemic disorder were excluded. Instrumentation for all studies was done using similar protocols. Heart rate is measured using a three-lead electrocardiogram (ECG) (SpaceLab Medical Inc., Issaquah, WA). A transcranial Doppler system (MultiDop X4, DWL Neuroscan Inc. Sterling VA) was used to obtain continuous measurements of blood flow velocity in the middle cerebral artery. The data was acquired by insonating the artery through the temporal windows using a $2-\mathrm{MHz}$ pulsed Doppler probe. The probe was positioned to record the maximal flow velocities and stabilized using a three-dimensional head frame positioning system. A photoplethysmographic device mounted on the middle finger of the non-dominant hand was used to obtain non-invasive beat-to-beat blood pressure (Finapres device, Ohmeda Monitoring Systems, Englewood, Colorado). To eliminate effects of gravity, the hand was held at the level of the right atrium and supported by a sling. All physiological signals were digitized at $500 \mathrm{~Hz}$ using Labview NINDAQ software (National Instruments, Austin, TX) and stored for offline analysis. Before data were analyzed they were down-sampled to $50 \mathrm{~Hz}$.

- STS protocol: After instrumentation, subjects sat in a straight-backed chair with their legs elevated at $90^{\circ}$ in front of them. After five minutes of stable recordings, the subjects were asked to stand up. Standing was defined as the moment both feet touched the floor, recorded by a force platform.

- HUT protocol: The subjects rested on their back in the supine position for 
10 minutes. After resting in this position, the table was then tilted to $70^{\circ}$ for 10 minutes.

- Syncope protocol: Using the HUT protocol described above the subjects were tilted to $70^{\circ}$ from supine to upright position and then kept upright until presyncope ( $>35 \mathrm{~min}$ ) at this time the subjects were returned to supine position, and the subjects regained consciousness immediately

The STS and the open loop model data analyzed were collected from Lewis A. Lipsitz and Vera Novaks laboratories at Hebrew Senior Life and at the Beth Israel Deaconess Medical Center, Boston, MA. All subjects provided informed consent approved by the Institutional Review Board at Hebrew Senior Life and at the Beth Israel Deaconess Medical Center. HUT data used for the syncope study was obtained by Jesper Mehlsen, Medical Director, Department of Physiology and Nuclear Medicine, Frederiksberg Hospital, University of Copenhagen, Denmark.

\subsection{Modeling strategy}

The following protocol provides an outline of the methodology that has guided our work with the development of patient specific models.

- Data, knowledge and structure: Development of reliable patient specific physiological models require detailed knowledge of the underlying biological system combined with a clear definition of the outcomes that the model is supposed to predict or prescribe. Sufficient insight into both the physiology and available modeling techniques is crucial. In this spirit, structures in the data analyzed in this study were revealed using standard statistical tools combined with nonlinear optimization. Results of these analysis lead to knowledge of the system revealed through collaboration between mathematicians and physicians. Other methodologies that can be used for analyzing the structure of the system include filtering and generalized principal component analysis.

- Canonical models: The level of details incorporated in the models reflect the system analyzed. In order to identify and estimate patient specific parameters in an effective and reliable way the number of parameters has to be kept as low as possible so all unimportant elements are excluded (the principle of parsimony). The models will be based on first principles (e.g., 
conservation laws) whenever possible and the parameters shall have a physiological interpretation. Such models are denoted canonical models. The models discussed in this review were developed to study patient specific short-term regulation of heart rate, blood pressure, and cerebral blood flow velocity during STS and HUT. The models were formulated as dynamical systems. Steady state analysis of the model equations were considered. Afterward models were used for analysis of baseline dynamic behavior and later coupled with control models allowing prediction of dynamics during the orthostatic challenges (STS and HUT).

- Parameter identification and estimation: The main goal with the models presented here $[27,28,30,21,20,22,3,31,5,2]$ was to identify biomarkers (model parameters) that allow the models to display the dynamic behavior observed in the data. Estimation of these quantities requires solution of an inverse problem, where a set of parameters is estimated that minimize the error between measured and computed quantities. For example, in the open loop heart rate model, a set of parameters minimizing the least squares error between computed and measured values of heart rate was identified, and in the closed loop model we minimized the least squares error between computed and measured values of blood flow velocity and arterial blood pressure.

For a given model and a given set of data, it is likely that the model is insensitive to a subset of the model parameters, i.e., changes in that subset of parameters has a negligible impact on the solution. This set of parameters is called insensitive, and such parameters cannot be estimated via solution to the inverse problem. Second, among the subset of parameters that are sensitive it is likely that parameters are correlated. For example, two resistors in series could both be sensitive, but it is not possible to identify both resistors separately, knowing only the total potential drop and current, e.g., $R_{T}=R_{1}+R_{2}=5$ have indefinitely many solutions for $R_{1}$ and $R_{2}$. The latter problem is addressed in some of our recent studies [31, 5] using a technique called subset selection. Once a set of sensitive uncorrelated parameters have been identified and estimated, statistical methods should be invoked to compare parameters within and between groups of individuals. In the models $[17,21,31]$ we used analysis of variance (ANOVA) to show if the limited set of model parameters vary between healthy young and healthy elderly subjects. To understand patient specific behavior, model parameters and initial conditions (considered as a special kind of parameters) were es- 
timated using literature data coupled with anthropometric (height, weight, age, gender) information, allometric scaling relations, as well as mean and steady state values extracted from the data.

- Optimization algorithms: To perform the required parameter estimations non-linear optimization algorithms was used. Two methods were used, gradient free methods: the Nelder-Mead method (a simplex method) [18, 20, 21, 22], genetic algorithms [7], and implicit filtering [7]; and gradient-based methods: Newtons method and the Levenberg-Marquardt method [31, 5]. The advantage of gradient-based methods is that sensitivities are computed as part of the optimization. A disadvantage of these methods is that the ODEs should be differentiated with respect to each of the parameters. Other methods that have received much attention recently include Kalman filtering [3], particle filters, functional differential analysis, and sequential Monte Carlo (SMC) methods.

- Validation of models: The process of solving the inverse problem estimating parameters that allow the models to predict data is one aspect of model validation, but this type of analysis should be combined with more general validation methods. For example, once a set of parameters have been estimated using one dataset, the models should be validated using other datasets not used for the parameter estimation. However, this type of validation often cannot be used for biological systems where inter and intra variations within and between groups of individuals are large. One way to validate such models is to use K-fold cross-validation [13] where a subset within one data-set is used for prediction of model parameters, while the remaining data are used for validation. Other validation methodologies include model reduction (discussed in [6]) and analysis of sub-mechanisms. Common for all of these validation methods is that if a model fails to be validated it should be adjusted. This process of iterative model development often generates important new insights into the underlying physiology [12, 1, 40, 30].

- Biomarkers: Patient specific model parameters estimated using reliable optimization techniques from well-validated models have potential to be used as biomarkers. This requires that confidence intervals for each identified parameters are small and that some certainty is achieved that the optimized parameters are not a result of optimization methods terminating at some local minimum. This type of methodology using models to estimate biomarkers 
can provide insight into quantities that cannot directly be measured experimentally. Examples include estimation of cerebrovascular resistance [17, 31], delay in baroreflex firing rate, baroreflex dampening, and baroreflex gain $[21,22]$. To determine if biomarkers differ significantly between groups of subjects, and if biomarkers can be used to identify variant causes of a given illness statistical tests can be performed. For example, in a recent study [31] we showed that cerebrovascular resistance is increased with aging.

- Multiscale models: Frequently models contain several scales, one could have an overall system level model predicting overall pressure level coupled with a detailed 3D model describing wave propagation and blood flow dynamics in a given artery. Coupling of such models have received much attention recently, and it is important when models are coupled that the overall dynamics is preserved. For example if a given biomarker has been identified from a system level model, if modeled correctly, the detailed model should serve to refine prediction of the biomarker.

We emphasize that the variant steps discussed above are not all independent, and should merely be considered as components important to assess in development of subject specific models. Overall, the main components included are: choice of model, parameter identification, parameter estimation, model validation, and comparison of outcomes (biomarkers) among study populations.

\subsection{Mathematical models}

Two models will be discussed an open-loop model predicting baroreflex mediated changes in heart rate in response to changes in blood pressure (provided as an input to the model) and a closed-loop model predicting baroreflex and cerebral autoregulation of arterial (finger) blood pressure and cerebral blood flow velocity in response to changes in heart rate (provided as an input to the model). Both models have been used for "model based" data analysis using input quantities defined apriori to predict model parameters (biomarkers) that minimized the error between measured and computed values of output quantities. In addition to the biomarkers, this approach also allow prediction of internal quantities, which cannot be measured experimentally. We like to view our model based data analysis as an in silico investigation of the feedback mechanisms, allowing inaccessible parts

to be made accessible (through the model predictions). For the open loop heart 
rate model, we used blood pressure data as an input to predict heart rate and the links made visible through the model include baroreflex firing rate, sympathetic and parasympathetic tone, and concentrations of acetylcholine and noradrenaline, and for the closed loop model quantities predicted include cerebrovascular resistance, cerebral and systemic blood pressure. These model based quantities can be used to form hypotheses for how these "invisible" quantities may vary, and with more experiments, it may be possible to validate the various sub-models. Thus, with sufficient validation, we may view the model together with the outcomes as a method that can provide insight into an individuals control system like a fingerprint. Such biomarkers have potential to be relevant for treatment of several diseases such as hypertension, see [20,18].

\subsubsection{Open loop model}

The overall function of the baroreceptor feedback mechanism is known. However, the underlying biochemical mechanistic processes are not fully understood and are difficult to investigate in-vivo. In the studies summarized here we used STS and HUT experiments to investigate the short-term baroreceptor feedback regulation of heart rate, detailed description of the model can be found in [24, 25, 30, 21, 22]. To investigate baroreflex regulation of heart rate we developed the model shown in Fig. 4. This model uses blood pressure as an input to predict heart rate using the following five steps:

- Mean blood pressure is used to predict afferent baroreflex firing rate.

- Sympathetic and parasympathetic outflows are computed from the baroreflex firing rate combined with stimulation by muscle sympathetic nerves, the vestibular system, and from central command.

- Concentrations of acetylcholine and noradrenaline are computed as functions of the sympathetic and parasympathetic outflow.

- Heart rate potential is computed from chemical concentrations.

- Heart rate is predicted from the heart rate potential.

The baroreflex model uses weighted mean blood pressure as an input to predict the afferent firing rate using a nonlinear differential equation of the form

$$
\begin{aligned}
\frac{d n_{i}}{d t} & =k_{i} \frac{d \bar{p}}{d t} \frac{n(M-n)}{(M / 2)^{2}}-\frac{n_{i}}{\tau_{i}}, \quad i=S, I, L \\
n & =n_{S}+n_{I}+n_{L}+N,
\end{aligned}
$$




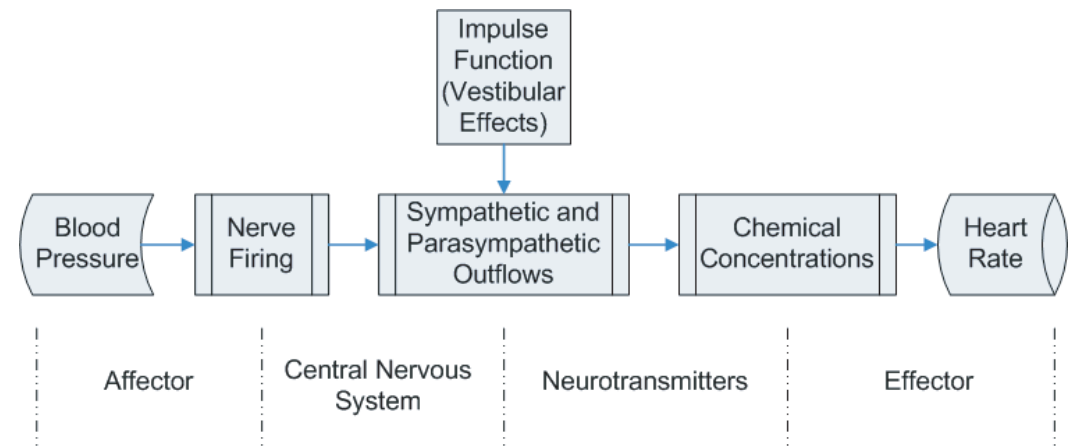

Figure 4: Elements of the baroreceptor feedback chain controlling heart rate.

where $n_{i}[1 / \mathrm{sec}]$ denotes the firing-rate and $k_{i}[1 / \mathrm{mmHg}]$ denotes the gain of the stimulus displayed by the neuron of type $i$ (three types of neurons are included fast or short time-scale term $S$, intermediate $I$, and slow or long time-scale term $L)$. $N[1 / \mathrm{sec}]$ denotes the baseline firing rate, $M[1 / \mathrm{sec}]$ denotes the maximum firing rate, and $n[1 / \mathrm{sec}]$ denotes the integrated firing rate. This model accounts for hysteresis observed between increases and decreases in mean pressure [25]. If the change in pressure is negative (as opposed to positive), the two terms in (1) have the same sign leading to a larger net change in firing rate.

The baseline firing-rate $\mathrm{N}$ cannot exceed the maximum firing-rate $(N<M)$ and we assume that $N><M / 2$. To enforce these bounds we have parameterized $N$ using a sigmoidal function of the form

$$
N=\frac{M}{2}+\frac{\eta^{2}}{1+\eta^{2}}\left(M-\frac{N}{2}\right)
$$

where $\eta$ is the unknown parameter to be identified.

The baroreflex firing rate model depends on the weighted mean pressure [mmHg] computed as

$$
\bar{p}(t)=\int_{-\infty}^{t} e^{-\alpha s} p(s) d s, \quad \text { or equivalently } \quad \frac{d \bar{p}}{d t}=\alpha(-\bar{p}+p),
$$

where $\alpha$ is the weight. A large value of $\alpha$ [1/sec] gives rise to a small weight of the past time (short memory), while a small value of $\alpha$ indicates larger weight (long memory). Thus, the weighted mean pressure $\bar{p}$ is a function of time, which oscillates with the same frequency as the instantaneous pressure $p$, but with smaller amplitude. For the studies analyzed here we let $\alpha=1$. 
The afferent firing rate $n$ is used for prediction of sympathetic $T_{\text {sym }}$ and parasympathetic $T_{p a r}$ outflows. Parasympathetic outflow is proportional to the firing rate, while the sympathetic outflow is inversely proportional to the firing rate and is dampened (with rate $\beta$ ) by the parasympathetic outflow. In addition, sympathetic outflow is modulated through activation via central command, the vestibular system, and via MSNA. The latter is lumped into the contribution $u(t)$ defined using an impulse function. Thus,

$$
\begin{aligned}
& T_{\text {par }}=\frac{n(t)}{M}, \quad T_{\text {sym }}=\frac{1-n\left(t-\tau_{d}\right) / N+u(t)}{1+\beta T_{p a r}}, \quad \text { where } \\
& u(t)=-\left(b\left(t-t_{m}\right)\right)^{2}+u_{0}, \quad b=\sqrt{\frac{4 u_{0}}{t_{p e r}^{2}}}, \quad \text { and } \quad t_{m}=t_{s t}+\frac{t_{p e r}}{2},
\end{aligned}
$$

where $\tau_{d}$ [sec] denotes the delay of the sympathetic response, $u_{0}$ (dimensionless), $t_{s t}$ [sec], and $t_{\text {per }}$ [sec] denote the magnitude and timing of the MSNA/central command/vestibular stimulation.

Using the sympathetic and parasympathetic outflows, nondimensionalized concentrations of acetylcholine $C_{a c h}$ and noradrenaline $C_{n o r}$ were computed using the first order equation

$$
\frac{d C_{i}}{d t}=\frac{-C_{i}+T_{j}}{\tau_{i}}, \quad i=\text { nor, ach } \quad \text { and } \quad j=\text { sym,par. }
$$

Parameters in this equation include characteristic time scales for noradrenaline and acetylcholine, $\tau_{\text {nor }}$ and $\tau_{\text {ach }}$ [sec ]. In this equation we have lumped the long chain of biochemical reactions into a first order reaction equation and taken the accumulated release times $\tau_{i}$ to be equal to the average clearance and consumption time for the respective substances.

The heart rate potential $\phi$ [beats] was computed using an integrate and fire model of the form

$$
\frac{d \phi}{d t}=H_{0}\left(1+M_{S} C_{n o r}-M_{P} C_{a c h}\right)
$$

where $H_{0}$ denotes intrinsic heart rate, which we predicted as a function of age $\left(H_{0}=118.1-0.57 \times\right.$ age $\left.[10,23]\right)$. The remaining parameters $M_{S}$ and $M_{P}$ represent the strength of the response to changes in the concentrations. To bound heart rate within physiological values, we constrained $M_{S}$ and $M_{P}$ in the interval $[0,1]$. This was done by introducing the parameters $\zeta_{S}$ and $\zeta_{P}$ that fulfill

$$
M_{S}=\frac{\zeta_{S}^{2}}{1+\zeta_{S}^{2}} \quad \text { and } \quad M_{S}=\frac{\zeta_{P}^{2}}{1+\zeta_{P}^{2}} .
$$


When $\phi$ reaches 1 it is reset to 0 , and heart rate is computed as inverse of the interval $\phi=0$ to $\phi=1$, i.e.,

$$
\mathrm{HR}=1 /\left(t_{\phi=1}-t_{\phi=0}\right)
$$

summary this model can be written on the form

$$
\begin{aligned}
\frac{d \bar{x}}{d t} & =f(\bar{x}, \xi(t), \bar{p}(t) ; \theta) \\
\bar{x} & =\left\{n_{i}, C_{a c h}, C_{n o r}, \phi\right\}, \quad i=S, I, L \\
\theta & =\left\{k_{i}, \tau_{i}, M, \eta, \tau_{d}, \beta, t_{s t}, t_{p e r}, u_{0}, \tau_{a c h}, \tau_{n o r}, \zeta_{S}, \zeta_{P}\right\}, \quad i=S, I, L \\
\xi & =\left\{n, T_{\text {sym }}, T_{\text {par }}\right\}
\end{aligned}
$$

where $\bar{x}(t)$ denotes the states, $\xi(t)$ the auxiliary equations, $\bar{p}(t)$ denotes the mean pressure (input from data), and $\theta$ denotes the model parameters.

This model is validated against heart rate, i.e., with this model we seek to identify a set of parameters $\theta$ that minimize the least squares error

$$
J=r^{T} r, \quad \text { where } \quad r=\left|y_{c}-y_{d}\right| .
$$

The output $y$ is the heart rate, i.e., $y_{c}=\mathrm{HR}_{c}=f(x, \theta)$ denotes computed values of heart rate and $y_{d}=\mathrm{HR}_{d}$ denote the heart rate data.

\subsubsection{Closed loop model}

The model discussed above, predicted regulation of heart rate as a function of blood pressure. However, data analyzed also include measurements of cerebral blood flow velocity. Therefore, in another series of studies [18, 20, 22, 31, 5] we developed a closed loop model predicting autonomic (baroreflex mediated) and cerebral autoregulation of arterial blood pressure and cerebral blood flow velocity using heart rate as an input. The most general form of the closed loop model is shown in Fig. 3. This model includes the systemic circulation, including the left heart (the atria and the ventricle) the aorta and vena cava, arteries and veins in the upper and lower torso as well as arteries and veins in the legs.

The dotted lines on the figure indicate the diaphragm movement during respiration: the movement of the diaphragm change the trunk pressure and the transmural pressure of the vessels in the chest region. To model this we let

$$
p_{\text {ext }}(t)=\frac{A_{u}}{2}\left(\cos \left(\frac{2 \pi t}{T_{\text {insp }}}\right)-1\right)+B_{u},
$$


where $A_{l}$ is the amplitude, $B_{l}$ the base level value, and $T_{i n s p}$ is the duration of inspiration. Similarly organs below the diaphragm, e.g. the liver, experiences changes in transmural pressure. However, the later blood pressure change is phase shifted by 180 degrees compared to that in the chest region. This is imposed to the model by allowing the exterior pressure $p_{\text {ext }}$ below diaphragm to vary as

$$
p_{\text {ext }}(t)=\frac{A_{l}}{2}\left(\cos \left(\frac{2 \pi t}{T_{\text {insp }}}\right)-1\right)+B_{l},
$$

where similar to the model discussed aobve $A_{l}$ is the amplitude, $B_{l}$ the base level value, and $T_{i n s p}$ is the duration of inspiration. To shift the two signals by $180^{\circ}$ we changed the sign of $A_{u}$ and $A_{I}$.

Finally, the period after inspiration has ended and during expiration we let $p_{\text {ext }}(t)=B_{l}$. Typically, the length of the respiration cycle is $T_{\text {resp }}=8 / 3 T_{\text {insp }}$.

In fact, this frequency and the depth of respiration are controlled. However, for the studies analyzed here, the subjects were asked to breathe to a metronome at a uniform depth, thus the model proposed above is adequate. If one has airflow data, it is possible to model exterior pressure directly as a function of airflow velocity, this approach was used in [4].

The closed loop model consists of three pars: A cardiovascular model predicting arterial blood flow and pressure in the various compartments; an autonomic regulation model predicting control of vascular tone (resistance and compliance), heart rate and cardiac contractility; and a cerebral autoregulation model predicting cerebrovascular resistance.

\section{Cardiovascular Model}

Each compartment in this model consists of a collection of arteries and veins of same caliber all with approximately the same pressure. Exceptions are the two compartments representing the left ventricle and atrium. Flow in this model is computed using an analogy to an electrical network with resistors and capacitors. Using this terminology, flow between compartments are analogous to current, pressure of each compartment is analogous to voltage, and compliance of each compartment is analogous to capacitance, while the resistance is the same in both formulations. Following this analogy the volume of each compartment is related to the pressure according to

$$
V-V_{\text {unstr }}=C\left(p-p_{\text {ext }}\right),
$$

where $V[\mathrm{ml}]$ is the total volume of the compartment, $V_{\text {unstr }}[\mathrm{ml}]$ is the unstressed volume, $C[\mathrm{ml} / \mathrm{mmHg}]$ is the compliance, $p[\mathrm{mmHg}]$ is the blood pressure, and 


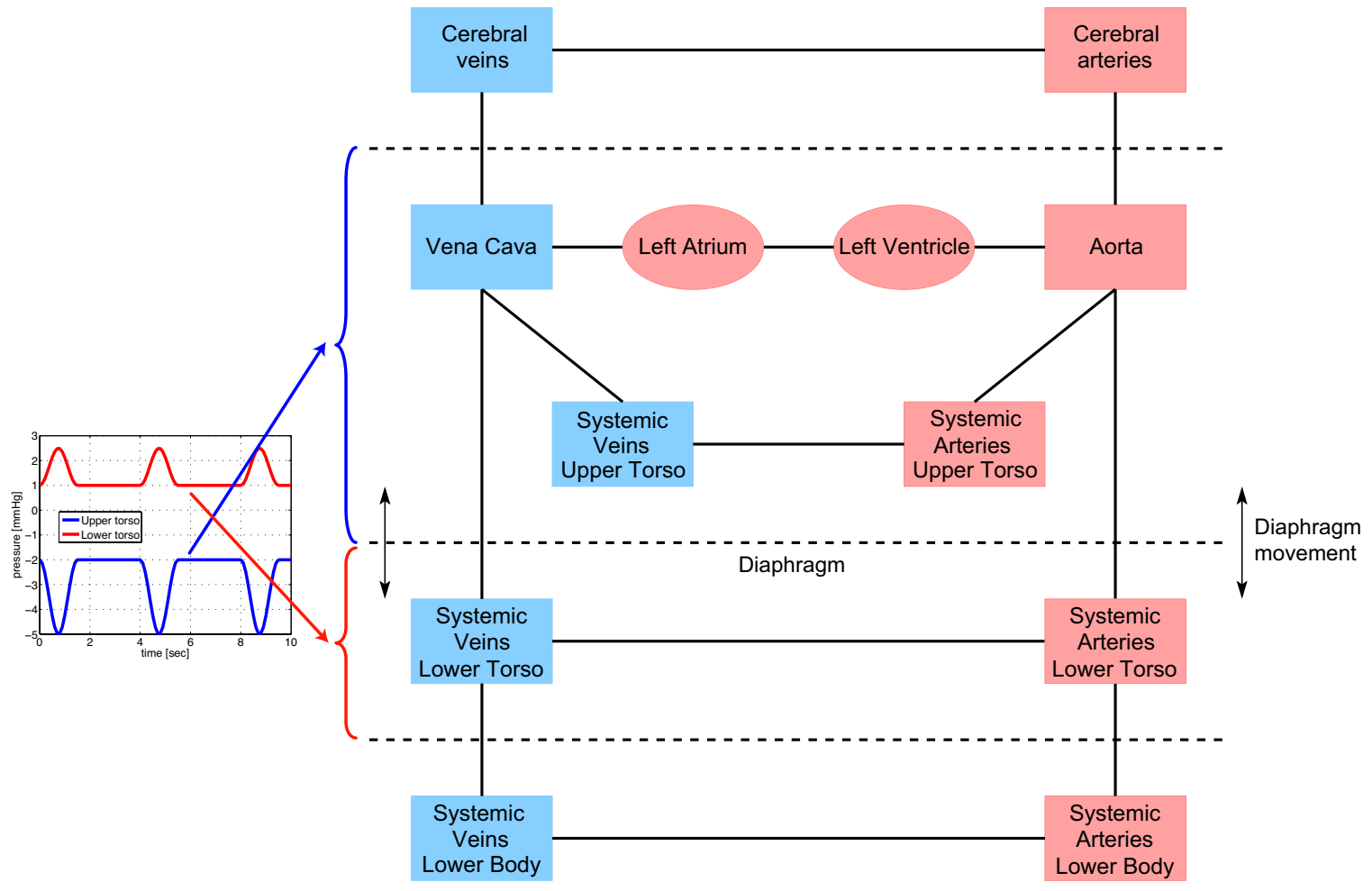

Figure 5: Diagram of the closed loop model. Arteries and ventricle are red and veins are blue. The influences of the diaphragm movement on the exterior vessel pressure is shown to the left. 
$p_{\text {ext }}[\mathrm{mmHg}]$ is the pressure of the tissue immediately outside the compartment which changes due to change in postural position or due to respiration. For compartments in the legs and brain we assumed that the external pressure $p_{\text {ext }}$ is constant, while for the compartments in the upper and lower torso the external pressure is modulated by movement of the diaphragm. Flow between compartments are computed using Ohms law which state that

$$
q=\frac{p_{\text {in }}-p_{\text {out }}}{R},
$$

where $q[\mathrm{ml} / \mathrm{sec}]$ denote the flow, $p_{\text {in }}$ and $p_{\text {out }}[\mathrm{mmHg}]$ are the pressures of the two compartments, and $R$ [ml/sec $\mathrm{mmHg}]$ is the resistance to flow. Differentiating (7) gives

and using (8) we get

$$
\frac{d V}{d t}=C \frac{d\left(p-p_{e x t}\right)}{d t}+\left(p-p_{e x t}\right) \frac{d C}{d t}
$$

$$
C \frac{d p}{d t}=C \frac{d p_{e x t}}{d t}-\left(p-p_{e x t}\right) \frac{d C}{d t}+q_{i n}-q_{\text {out }} .
$$

A differential equation of this form can be derived for all arterial and venous compartments. Note, for steady state simulations we assumed that $p_{e x t}=0$ and $C$ is constant. In general, $p_{\text {ext }}$ should be modulated with respiration (e.g., as suggested in (5) and (6) and $C$ should be controlled, or as discussed in several studies by Ursino et al. [41, 42, 43] it may be appropriate to model $C$ using a nonlinear function of the stressed volume.

For compartments representing the left heart (the left and right atrium) two different models have been analyzed. One model proposed by Ottesen [28] uses a generalized activation function to predict the pressure in the heart compartments; the other is a simple elastance model. The advantage of the elastance model is that it contains only 4 parameters, while the more accurate model by Ottesen contain 14 parameters. The Ottesen model predicts the left heart pressure as

$$
\begin{aligned}
p_{l h} & =a(V(t)-b)^{2}+(c(t) V(t)-d) f(\tilde{t}) / f\left(t_{p}\right), \\
f(\tilde{t}) & =\left\{\begin{array}{cc}
p_{p} \frac{\tilde{t}(\beta-\tilde{t})^{m}}{n^{n} m^{m}\left(\beta /(m+n)^{m+n}\right.} & 0 \leq \tilde{t} \leq \beta \\
0 & \beta \leq \tilde{t} \leq T_{i}
\end{array},\right.
\end{aligned}
$$

where $a\left[\mathrm{mmHg} / \mathrm{ml}^{2}\right]$ is related to the elastance during relaxation, $b[\mathrm{ml}]$ represents the volume at zero diastolic pressure, $c(t)[\mathrm{mmHg} / \mathrm{ml}]$ represents contractility (note during steady state $c$ is constant), and $d[\mathrm{mmHg}]$ is related to the volumeindependent component of the developed pressure. In the activation function $f, T_{i}$ 
[sec] denotes the length of the ith cardiac cycle, $\beta$ [sec] denotes the onset of relaxation, $n$ and $m$ characterize the contraction and relaxation phases and $p_{p}[\mathrm{mmHg}]$ is the peak value of the activation. The ability to vary heart rate is included in the pressure equation by scaling the time $t_{p}$ [sec] and peak values $p_{p}$ of the activation function $f$, for details see [28]. An advantage of this model is that the ejection effect is easily incorporated, i.e., the fact that dynamical changes in ventricular volume due to ejection of blood affect the ventricular contractility. Thus the model is suitable when varying afterloads are considered. The corresponding elastance model is given by

$$
\begin{aligned}
p_{l h} & =E(\tilde{t})\left(V(t)-V_{d}\right) \\
E(\tilde{t}) & =\left\{\begin{array}{cc}
\left(E_{M}-E_{m}\right)\left(1-\cos \left(\frac{\pi \tilde{t}}{T_{M}}\right)\right) & 0 \leq t \leq T_{M} \\
\left(E_{M}-E_{m}\right)\left(\cos \left(\frac{\pi\left(\tilde{t}-T_{M}\right)}{T_{R}}\right)\right) & T_{M} \leq \tilde{t} \leq T_{M}+T_{R} \\
0 & T_{M}+T_{R} \leq \tilde{t} \leq T_{i}
\end{array}\right.
\end{aligned}
$$

where $V_{d}[\mathrm{ml}]$ denote the volume at zero end-systolic pressure. In the elastance function $E, T_{M}$ and $T_{R}$ [sec] denote the time for maximum (systolic) elastance $\left(T_{M}\right)$ and the remaining time to relaxation $\left(T_{R}\right)$ and $E_{M}$ and $E_{m}[\mathrm{mmHg} / \mathrm{ml}]$ denotes the maximal (systolic, $\left.E_{M}\right)$ and minimal $\left(E_{m}\right)$ diastolic elastance. This model accounts for varying heart rate by defining scaled parameters $T_{M f}=T_{M} / T_{i}$ and $T_{R f}=T_{R} / T_{i}$. As before $T_{i}$ [sec] denotes the length of the current cardiac cycle. For either of the two models, a differential equation for the heart compartments can be obtained from conservation of volume, i.e., we let

$$
\frac{d V}{d t}=q_{\text {in }}-q_{\text {out }}
$$

where as before the flows are computed using Ohms law. It should be noted, that both the atrium and the ventricle are modeled using the same type of equations, but that parameters for the two heart chambers vary. Finally, the left ventricle cannot function without heart valves. In all studies summarized here, we used time varying resistances to represent the valves. These are defined such that a closed valve is represented by a high resistance $R_{\text {valve }, c}$ and an open valve is represented by a very low resistance $R_{\text {valve,o }}$. This can be done by defining valve resistances as

$$
R_{\text {valve }}=\min \left(R_{\text {valve }, o}-e^{-k\left(p_{\text {in }}-p_{\text {out }}\right)}, R_{\text {valve }, c}\right),
$$

where $k[1 / \mathrm{mmHg}]$ is a rate constant denoting the time it takes for the valve to close. 
Similar to the open loop model, the closed loop steady state model (i.e., no parameters are controlled $C, R, c$ are constant parameters) shown in Fig. 3 can be represented by a system of differential equations of the form coupled with a set of auxiliary equations

$$
\begin{aligned}
\frac{d \bar{x}}{d t} & =f\left(\bar{x}, \xi(t), T_{i} ; \theta\right), \\
\bar{x} & =\left\{p_{i}, p_{i, c}, p_{i, u t}, p_{i, l t}, p_{i, l}, V_{l a}, V_{l v}\right\}, \quad i=a, v \\
\theta & =\left\{R, C, a, b, c, d, n, m, T_{m f}, T_{M f}, E_{M}, E_{m}, T_{i}\right\}, \\
\xi & =\left\{R_{\text {valve }}, f, E, p_{l v}, p_{l a}\right\},
\end{aligned}
$$

where $\bar{x}(t)$ denotes the states, $\xi(t)$ denotes the auxiliary equations, $T_{i}$ denote the length of each cardiac cycle (input from data), and $\theta$ denote the model parameters.

This model is validated using measurements of heart rate (input), arterial blood pressure and blood flow velocity, i.e., with this model we seek to estimate a set of parametres $\theta$ that minimize the least squares error

$$
J=r^{T} r, \quad \text { where } \quad r=\left|y_{c}-y_{d}\right| .
$$

The output $y$ is a vector concatenating blood pressure and blood flow velocity, i.e., $y_{c}=\left\{p_{c}\left(t_{1}\right), p_{c}\left(t_{2}\right), \ldots, p_{c}\left(t_{N}\right), v_{c}\left(t_{1}\right), v_{c}\left(t_{2}\right), \ldots, v_{c}\left(t_{N}\right)\right\}=f(x, \theta)$ denotes computed values and $y_{d}=\left\{p_{c}\left(t_{1}\right), p_{c}\left(t_{2}\right), \ldots, p_{c}\left(t_{N}\right), v_{c}\left(t_{1}\right), v_{c}\left(t_{2}\right), \ldots, v_{c}\left(t_{N}\right)\right\}$ denote the corresponding data.

\section{Modeling sitting to standing}

To allow the model to predict blood pressure and cerebral blood flow velocity dynamics during postural change from sitting to standing we incorporated changes in hydrostatic pressure to allow pooling of the blood in the legs. To do so we modified equations predicting flow to and from the upper to the lower body as

$$
\begin{aligned}
q & =\frac{\left(p_{\text {in }}-\rho g h_{\text {in }}\right)-\left(p_{\text {out }}-\rho g h_{\text {out }}\right)}{R}, \\
h(t) & =\frac{h_{M}}{\left.1+e^{-k\left(t-T_{\text {up }}-\delta\right.}\right)}
\end{aligned}
$$

where $T_{u p}$ [sec] is the time at which the subject stands up, $h_{M}[\mathrm{~cm}]$ is the maximum height needed for the mean arterial pressure to drop as indicated by the data, and $\delta$ [sec] is the latency for the transition to standing. This idea is naturally generalized to cover head up tilt too. 


\section{Modeling autonomic regulation}

Only one of our previous studies [20] modeled autonomic regulation. In this study we assumed that cardiac contractility $c[\mathrm{mmHg} / \mathrm{ml}]$ and systemic peripheral resistances $R[\mathrm{ml} / \mathrm{sec} \mathrm{mmHg}]$ in the upper body and the legs were increased in response to the drop in arterial pressure, while compliance $C[\mathrm{ml} / \mathrm{mmHg}]$ was decreased. Inspired by [27] we used a first order set-point equation to model this control.

$$
\begin{aligned}
\frac{d x}{d t} & =\frac{-x+x_{c t r}\left(p_{a}\right)}{\tau}, \\
x_{c t r}\left(p_{a}\right) & =\left(x_{M}-x_{m}\right) \frac{\alpha^{k}}{p_{a}^{k}+\alpha^{k}}+x_{m}, \quad x=R, c \\
x_{c t r}\left(p_{a}\right) & =\left(x_{M}-x_{m}\right) \frac{p_{a}^{k}}{p_{a}^{k}+\alpha^{k}}+x_{m}, \quad x=C .
\end{aligned}
$$

In the above equation $x_{c t r}$ is an increasing (for $R, c$ ) / decreasing $(C)$ sigmoidal function of arterial pressure. Using a sigmoidal function allows the system to display saturation beyond the limit of regulation. In this function $x_{M}$ and $x_{m}$ denote the upper and lower limit for the parameter controlled, the parameter $\alpha$ is set to ensure that $x(t)$ returns to the value of the controlled parameter found during steady state, and $k$ denotes the steepness of the sigmoid. Finally, the parameter $\tau$ characterizes the time it takes for the control to reach its maximal effect. In addition to the active control we modelled resistances between arterial compartments using a sigmoidal equation similar to the one given in (15).

It should be noted, that this direct control as a function of pressure is significantly simpler than the more complex baroreflex model presented earlier, the disadvantage is that this is a purely empirical model not accounting for any of the physiological mechanisms, known to be involved in the baroreflex regulation. Another important point is that the true baroreflex model includes a delay, which is not accounted for in the simpler model discussed above. However, if adequate parameters are found, one could couple the models and instead model the control using the baroreflex model described above.

\section{Modeling cerebral regulation}

Most models in the literature tempting to predict cerebral regulation are derived from models proposed by Ursino et al. [42, 41]. However, it is not clear if this model includes all possible mechanisms believed to be involved in cerebral regulation. Many factors have been proposed to play a role, including responses to changes in $\mathrm{CO}_{2}$ (often denoted as cerebral vasoregulation), responses to myogenic 
regulation (this is sometimes what is understood by the term cerebral autoregulation). Some recent studies have also indicated that a portion of cerebral regulation stems from neurogenic regulation. Initially, we attempted to model cerebral regulation using model similar to the set-point function proposed in (15) including only the myogenic aspects of the regulation. However, using this type of equation did not enable prediction of observed variation in cerebral blood flow velocity. Instead we used a open-loop control model formulated using a piecewise linear function with unknown coefficients to obtain a representative function that describes the time-varying response of the cerebrovascular resistance. To obtain such a function, we parameterized the cerebrovascular resistance using the piecewise linear function of the form

$$
\begin{aligned}
& R(t)= \sum_{i=1}^{N} \gamma_{i} H_{i}(t), \\
& H_{i}(t)=\left\{\begin{array}{cc}
\frac{t-t_{i-1}}{t_{i}-t_{i-1}} & t_{i-1} \leq t \leq t_{i} \\
\frac{t_{i+1}-t}{t_{i+1}-t_{i}} & t_{i} \leq t \leq t_{i+1} \\
0 & \text { otherwise }
\end{array}\right.
\end{aligned}
$$

where $\gamma_{i}[\mathrm{ml} / \mathrm{sec} \mathrm{mmHg}]$ are the unknown coefficients, which should be estimated together with the other parameters. Following the parameter estimation we used the predicted time-varying response to propose a cerebral regulation model. The most promising model analyzed had the form

$$
\begin{aligned}
R & =R_{\text {met }}+R_{\text {myo }}+R_{\text {neu }}, \\
\frac{d R_{\text {met }}}{d t} & =\frac{-R_{m e t}+\tilde{R}_{m e t}\left(q_{c}\right)}{\tau_{m e t}}, \\
\frac{d R_{\text {myo }}}{d t} & =\frac{-R_{\text {myo }}+\tilde{R}_{\text {myo }}\left(p_{a c}\right)}{\tau_{\text {myo }}}, \\
\frac{d R_{\text {neu }}}{d t} & =\frac{-R_{\text {neu }}+k_{\text {neu }} C_{a c h}\left(p_{a}\right)}{\tau_{\text {neu }}},
\end{aligned}
$$

where $R_{\text {met }}[\mathrm{ml} / \mathrm{sec} \mathrm{mmHg}]$ is the contribution from the metabolic regulation, $R_{\text {myo }}[\mathrm{ml} / \mathrm{sec} \mathrm{mmHg}]$ is the contribution from myogenic regulation, and $R_{\text {neu }}$ $[\mathrm{ml} / \mathrm{sec} \mathrm{mmHg}]$ is the contribution from neurally mediated control. Note, $R_{\text {met }}$ is modeled as a function of cerebral flow $q_{c}[\mathrm{ml} / \mathrm{sec}]$, while myogenic contribution is modeled as a function of cerebral arterial pressure $p_{c a}[\mathrm{mmHg}]$. Finally, the 


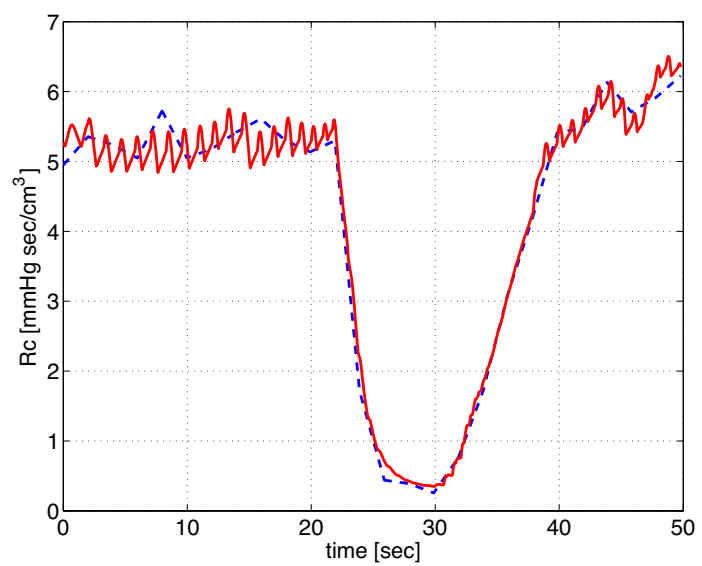

Figure 6: Cerebrovascular resistance predicted using a piecewise linear function (16) (blue) versus the control function (red) proposed in (17).

neurogenic contribution is modeled as a function of arterial pressure $p_{a}[\mathrm{mmHg}]$. It is believed that a potential neurogenic contribution to cerebral vasoregulation is both cholinergic and adrenergic in nature. Therefore we let the set-point equation use concentration of acetylcholine $C_{a c h}$ (dimensionless). The concentration of acetylcholine was computed similar to the open loop heart rate model, see equation (2). For the metabolic and myogenic contributions the control functions $\tilde{R}$ were sigmoidal functions similar to the one given in (15). Results of the two control models (17) and (16) are shown in Fig. 4. It should be noted that if we omitted any part of the proposed control function we were not able to reproduce the dynamic found from the piecewise linear function. One thing should be kept in mind is that both the spline model (16) and the differential equations model (17) has 26 parameters. Ideally, it would be better if the cerebral regulation model contained fewer parameters.

\subsection{Parameter estimation and model validation}

Many real life processes and systems can be modeled using non-linear ODE's or PDE's. A frequent difficulty in biomedical applications is that the model equations often have a large number of unknown parameters. For some systems it is possible to determine model parameters directly from the experimentally setup. However, in many cases it is difficult or even impossible to measure biomarkers, while other quantities can be measured. For example, during the STS and HUT experiments, 
typical measurements include arterial pressure and cerebral flow velocity.

To solve the inverse problem, one can invoke optimization techniques to estimate a set of model parameters that minimize the least squares error between computed and measured quantities. Whether the parameters for the mathematical model can be estimated assuming sufficient and error-free data is subject to an $a$ priori identifiability analysis. Two aspects typically have to be investigated: First using sensitivity analysis we are able to split model parameters in two sets include: "sensitive" and "insensitive" parameters. Sensitive parameters are characterized as parameters where variation in the parameter values invoke a significant change in the model output (see Fig. 7), while a change of insensitive parameters has a negligible impact on the model output. Second, among sensitive parameters, correlations can be present. This type of model dependencies can be predicted accurately for linear models, but for nonlinear models it is more difficult to analyze the system. Once a set of identifiable parameters have been identified, these parameters can be estimated using nonlinear optimization techniques. Following the approach put forward in $[6,31,5]$ we describe each of the three components in detail.

\section{Sensitivity analysis}

Sensitivities are computed with respect to output vector $y$ (heart rate for the open loop model summarized in (3) and blood pressure and blood flow velocity for the closed loop model summarized in (13)). For both of these models, the nominal parameter values range several orders of magnitude, e.g. for the small closed loop model analyzed in [31] the parameter $E_{m} \approx 0.05$, while $C_{v s} \approx 36$. To compute sensitivities more accurately, we scaled the parameters by the natural logarithm, i.e., the model input to the optimizer is given by $\tilde{\theta}=\ln (\theta)$.

Using the scaled parameters sensitivities can be computed as the change in the output variables with respect to the parameters, the absolute sensitivity is defined by

$$
S_{i, k}(t, \tilde{\theta})=\left.\frac{\partial y_{k}(t, \theta)}{\partial \tilde{\theta}_{i}}\right|_{\theta_{0}},
$$

where $\theta_{0}$, denotes the nominal values for the parameters. Even though parameters are scaled, quantities compared may still have different units (e.g. pressure and velocity in the closed loop model), thus it is appropriate to analyze relative sensitivities defined as

$$
S_{i k}(t, \tilde{\theta})=\left.\frac{\partial y_{k}(t, \theta)}{\partial \tilde{\theta}_{i}} \frac{\tilde{\theta}_{i}}{y_{k}(t, \theta)}\right|_{\theta_{0}}, \quad \tilde{\theta}_{i}, y_{k} \neq 0
$$


Note, for the open loop model $S_{i, k}$ is computed with respect to one output, heart rate, while for the closed loop model both pressure and velocity are concatenated together to provide one long output vector of length $2 N$, thus the length of $S_{i, k}$ is $2 N$. As discussed in $[6,4]$ the sensitivities can either be found using automatic differentiation, by setting up a set of analytical equations, or using finite differences. The finite difference approximation of the sensitivities is less accurate, but for most practical applications including those studied here, finite difference approximations provides sufficient accuracy. This should seen in the light of the high likelihood of introducing errors in analytical sensitivity calculations, in particular for models that has many parameters, e.g., if a model has two outputs and 21 parameters the system $2 \times 21=42$ sensitivity equations should be derived. An alternative approach is to use automatic differentiation to derive sensitivity equations, but these methods are computationally ineffective in particular if the differential equations are solved using Matlab. More efficient packages exist in C and Fortran, but these have not been analyzed for this study.

Using finite differences, the derivatives in the sensitivity equations can be computed using the forward difference approximation

$$
\frac{\partial y_{k}}{\partial \tilde{\theta}_{i}} \approx \frac{y_{k}\left(t, \tilde{\theta}+h e_{i}\right)-y_{k}(t, \tilde{\theta})}{h},
$$

where

$$
e_{i}=[0 \ldots 0 \widehat{1} \quad 0 \ldots 0]^{T}
$$

is the unit vector in the $i$ 'th component direction.

To rank the parameters from the most to the least sensitive, we used a scaled 2-norm to get the total sensitivity, $S_{i}$, to the $i$ 'th parameter

$$
S_{i}=\left(\frac{1}{2 N} \sum_{j=1}^{2 N} S_{i, k}^{2}\right)^{1 / 2} .
$$

Figs. 7 and 8 show examples of the sensitivity analysis. Fig. 7 shows ranking and time varying sensitivities for the open loop model using data from a healthy young subject. Note the change in solution arising from varying the insensitive parameter $\tau_{I}$ barely changes the model solution, while a significant change in heart rate is observed when the sensitive parameter $\zeta_{P}$ is varied. Fig. 8 shows the average ranking using data from 16 healthy young and elderly subjects computed from 
the closed loop model. The specific closed loop model used for these calculations included 4 vascular and one ventricular compartments, the model had 21 parameters.

It should be noted that the classical sensitivity analysis described above is a local analysis, and thus sensitivities depend on the values of the parameters. In this study the goal is to use sensitivity analysis to rank parameters in order of sensitivity and use this ranking in conjunction with results from subset selection to identify a set of parameters that can be estimated for all subjects. This is done prior to actual parameter estimations, thus the sensitivity ranking was computed using nominal parameter values.

In summary, results from the sensitivity analysis showed that both models include both sensitive and insensitive parameters. Sensitive parameters can often be estimated using optimization techniques, while insensitive parameters are difficult to estimate since a small change in the parameter value gives rise to a small change in the solution. This can become problematic when models are used to extract biological information from estimated parameter values as done in the studies summarized here. However, it should be noted that identifiability is a mathematical notion. For biological implications the precise values of parameters are not always important as long as they have certain characteristics, e.g., like being positive. An alternative method is to use generalized sensitivity analysis, which provide more insight into the dynamics of the model.

\section{Subset selection}

Subset selection can be approached using a number of methods as described in [32]. Below we outline the method used in [31]. In this study subset selection analyzes the Jacobian matrix $\left(r^{\prime}=d r / d \theta\right)$ computed from the residual vector $r$ (see equations (3) and (13)). The entry at row $i$ and column $j$ of the Jacobian is $\partial r_{i} / \partial \theta_{j}$. The Jacobian, singular value decomposition $r^{\prime}=U \Sigma V^{T}$ is used to obtain a numerical rank for $r^{\prime}$. This numerical rank is then used to determine $\rho$ parameters that can be identified given the model output $y$ defined in (3) and (13). QR decomposition is used to determine the $\rho$ identifiable parameters to which our system is sensitive as a group. This differs from sensitivity analysis, which finds parameters to which our system is individually sensitive. To estimate the number of uncorrelated parameters we used an error estimate in our computation of the Jacobian as a lower bound on acceptable singular values. For example, in the studies analyzed here we used Matlabs differential equations solver ODE15S with an absolute error tolerance of $10^{-6}$, i.e., the error of the numerical model solution is of order $10^{-6}$ and the error in the Jacobian matrix is approximately $\sqrt{10^{-6}}=10^{-3}$. 

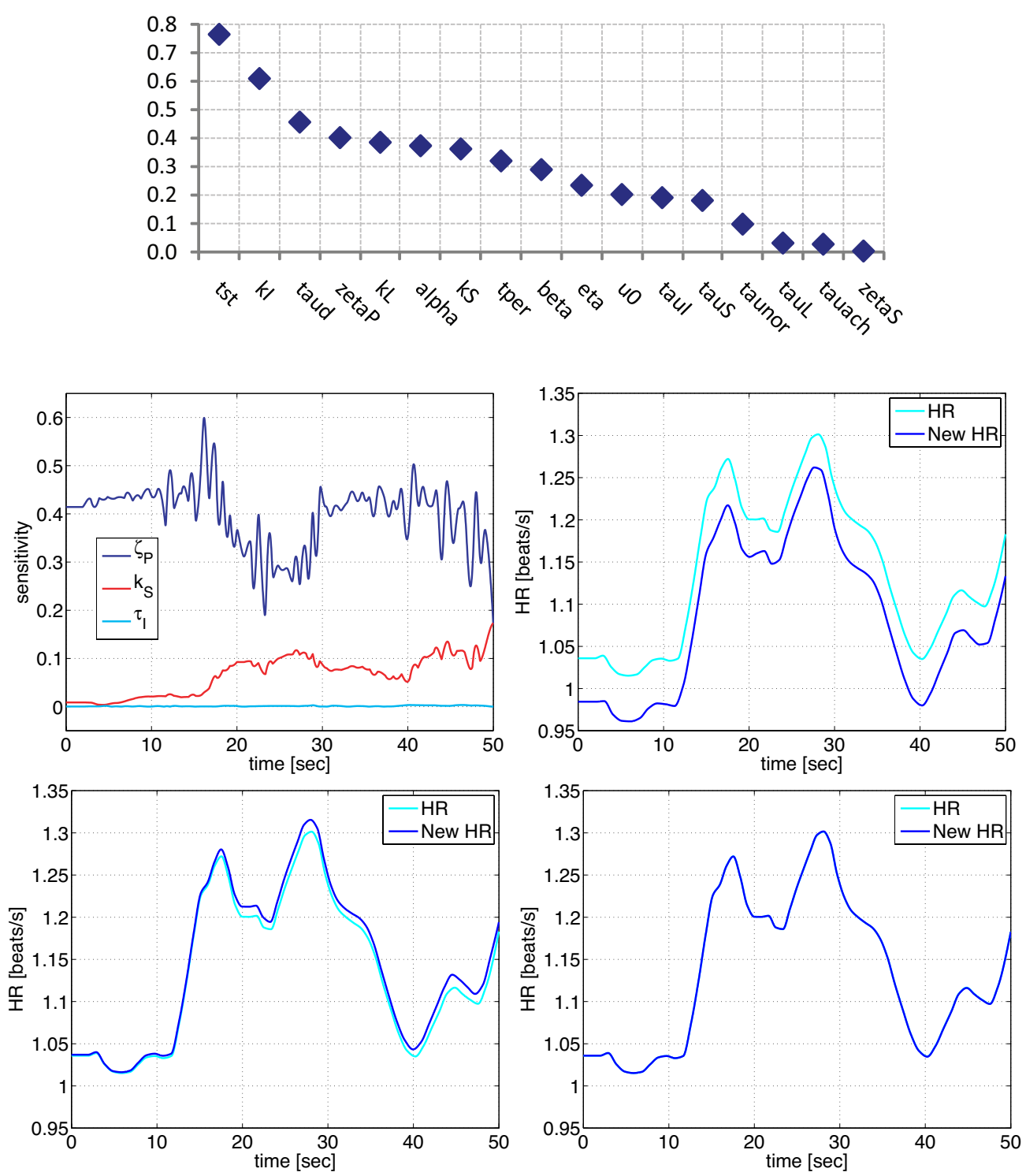

Figure 7: Sensitivity ranking for the open loop heart rate model. Sensitivities are computed with respect to heart rate for a healthy young subject. A weighted 2-norm was used to obtain the ranked sensitivities. Bottom panels show time varying sensitivities for a sensitive, an intermediate, and an insensitive parameter as well as the corresponding heart rates computed while varying the parameter in question. 


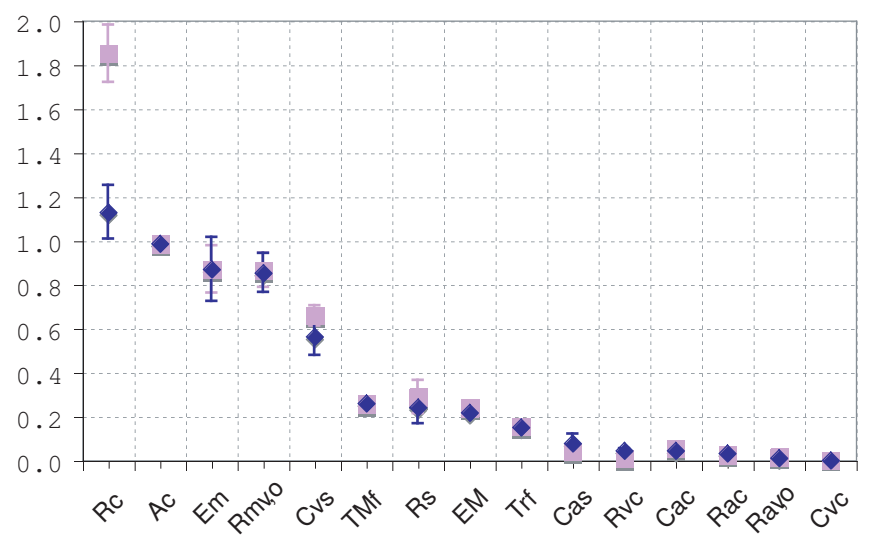

Figure 8: Sensitivity ranking for a 5 compartment (systemic arteries and veins, cerebral arteries and veins, and the left ventricle) closed loop model. Sensitivities are computed with respect to arterial pressure and cerebral blood flow velocity. A weighted 2-norm was used to obtain an average sensitivity over the entire time series.

Consequently, singular values should not be smaller than $10^{-3}$. Since the error of the Jacobian is an approximation, the smallest singular value that we accept is $10^{-2}$. Once the number of identifiable parameters has been determined, we find the most dominant parameters by performing a QR decomposition with column pivoting on the most dominant right singular vectors. The process begins by choosing the most sensitive parameter in a way similar but not identical to the sensitivity analysis of the previous section, the column with largest 2-norm is chosen. The algorithm chooses additional parameters in a way that keeps the condition number of the chosen columns small. Below we summarize subset selection method as an algorithm.

\section{Subset selection algorithm:}

1. Given an initial parameter estimate, $\theta_{0}$, compute the Jacobian, $r^{\prime}\left(\theta_{0}\right)$ and the singular value decomposition $r^{\prime}=U \Sigma V^{T}$, where $\Sigma$ is a diagonal matrix containing the singular values of $r^{\prime}$ in decreasing order, and $V$ is an orthogonal matrix of right singular vectors.

2. Determine $\rho$, the numerical rank of $r^{\prime}$. This can be done by determining a 
smallest allowable singular value.

3. Partition the matrix of eigenvectors in the form $V=\left[V_{\rho} V_{n-\rho}\right]$.

4. Determine a permutation matrix $P$ by constructing a $\mathrm{QR}$ decomposition with column pivoting, for $V_{\rho}^{T}$. That is, determine $P$ such that

$$
V_{\rho}^{T} P=Q R
$$

where $Q$ is an orthogonal matrix and the first $\rho$ columns of $R$ form an upper triangular matrix with diagonal elements in decreasing order.

5. Use $P$ to reorder the parameter vector $\theta_{0}$ according to $\hat{\theta}_{0}=P^{T} \theta_{0}$.

6. Make the partition $\hat{\theta}_{0}=\left[\hat{\theta}_{0, \rho} \hat{\tilde{\theta}}_{0, n-\rho}\right]$ where $\hat{\theta}_{0, \rho}$ contains the first $\rho$ elements of $\hat{\theta}_{0}$. Fix $\hat{\theta}_{n-\rho}$ at the a priori estimate $\hat{\theta}_{0, n-\rho}$.

7. Compute the new estimate of the parameter vector $\hat{\theta}$ by solving the reducedorder minimization problem

$$
\hat{\theta}=\arg \min _{\theta} J(\theta), \quad \text { with } \hat{\theta}_{n-\rho} \text { fixed at nominal values } \hat{\theta}_{0, n-\rho} \text {. }
$$

Fig 9 shows possible subsets of identifiable parameters from the same model that we used to show results of the sensitivity analysis. The model used for this study included 4 vascular and one ventricular compartments, the model had 21 parameters, and out of these only 4 could be estimated reliably given arterial blood pressure and cerebral blood flow velocity data measured during sitting. It should be noted that this model did not include any control mechanisms.

\section{Optimization techniques}

Sensitivity analysis and subset selection are examples of methods that can be used to address the question of parameter identifiability, other methods include Kalman filtering and principal component analysis. Common for these methods are that they identify a set of parameters and then using nonlinear optimization techniques it is possible to estimate the parameters in question. To do so we formulate a least squares problem (LQP) and seek to estimate a set of parameters that minimize the least squares errror between measured and computed quantities (as discussed in equations (4) and (14). In general, least squares problems (LQP) have several minima, because the problem has more than one solution or the error-function can 


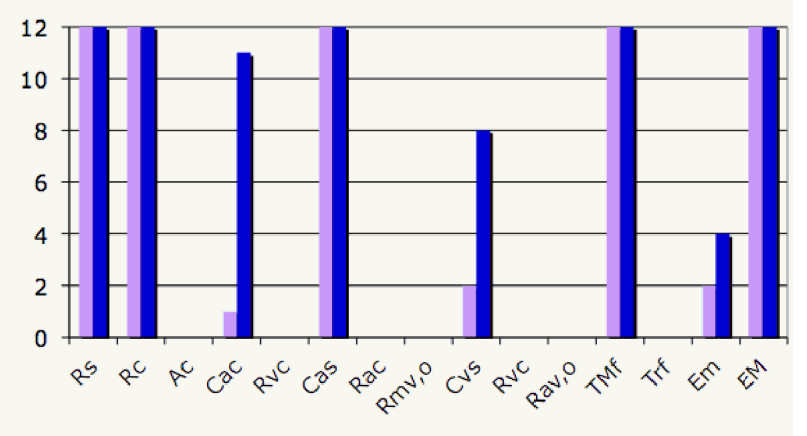

Figure 9: Subsets computed for 16 healthy young and 16 healthy elderly subjects using a 5 compartment closed loop model combined with arterial blood pressure and cerebral blood flow velocity data.

have several stationary points that do not correspond to the lowest value of the fitness landscape. Local search methods, like the Levenberg-Marquardt method, easily gets trapped in one of the local minima rather than finding the global minimum. To explore the whole search space one needs global search methods and if possible a physiological range for realistic values. Unfortunately, these methods converge very slowly once near a minimum. In contrast, gradient-based methods are efficient optimizers for nonlinear LQP's once a sufficiently good initial guess for the parameter values is available. Thus a recommendable strategy is to use the solutions from the global search as initial guesses for local optimization. In this way, one reduces the chance of missing the global minimum and the determination of all the minima is precise and fast. In the problems analyzed and discussed here we used the Nelder-Nead method (a gradient free global optimization method) to estimate parameters for the open loop model and a gradient based method (a Levenberg-Marquart method with Trustregions) for the closed loop model. Both methods worked well, but the Nelder-Mead method was significantly slower, while the Levenberg-Marquart method required that we estimated initial parameter estimates carefully.

In addition to estimation of model parameters, another important question is model validation. The studies analyzed here do not address this question. Methods typically used include K-fold cross validation [13], which use a part of the time series data for parameter estimation and another part for model validation. 


\section{Discussion}

Below we summarize and discuss result reported in $[24,25,30,18,19,20,21$, $22,6,31,5]$. We divide the presentation into three subsections; open loop model, closed loop model, and syncope.

\subsection{Open Loop model}

Results with the open loop model have been reported in [24, 25, 21, 22, 7] results were obtained with the STS and HUT protocol. In [21] we analyzed STS data from three groups of subjects including healthy young, healthy elderly, and hypertensive elderly healthy young subjects, and in [22] we analyzed both STS and HUT data from five young subjects. For both studies all model parameters were predicted using the Nelder-Mead optimization method. Results showed that standard deviations were typically high, however, for both studies we were able to detect interesting differences between the groups of subjects. In [21] (results
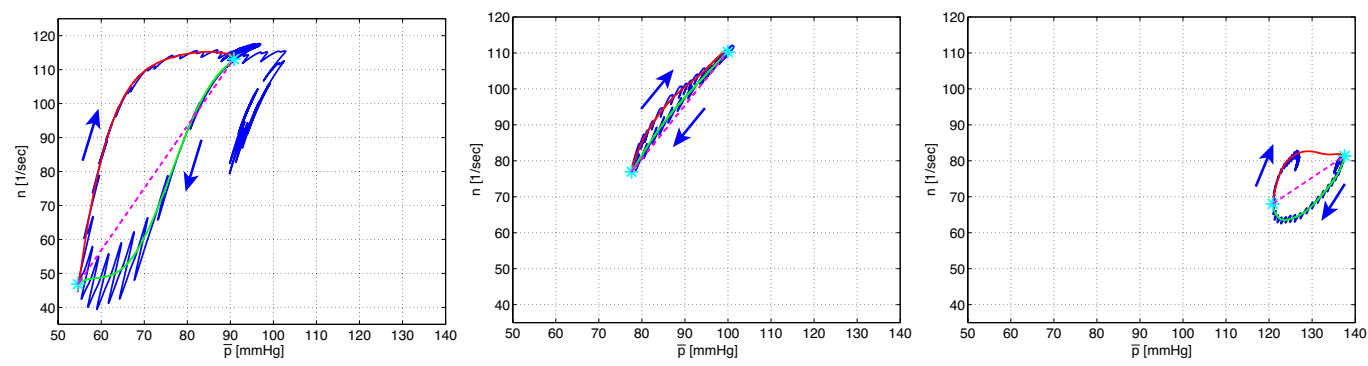

Figure 10: Example hysteresis depicting changes in baroreflex firing rate $(n)$ as a function of mean blood pressure $(\bar{p})$. Results are shown for a healthy young subject (left), a healthy elderly subject (center), and a hypertensive elderly subject (right). Dashed lines through the curves were used to determine the overall slope, and arrows indicate responses to change in pressure. Baroreceptor hysteresis is an important feature that demonstrates how baroreflex controls adapt in response to to differences in vascular compliance that occur with aging.

shown in Figs. 3 and 10) we observed that the hysteresis loop changed significantly with age: for health young subjects the hysteresis loop was significantly larger and the slope higher than for any of the other two groups, see Fig. 10. The healthy elderly subjects had a very narrow loop and mean pressures were higher than for the young subjects. Finally for the hypertensive subjects we noticed that 
for most subjects the hysteresis loop was not closed, indicating that within the timeframe included in the experiments the pressure does not return to the value obtained during sitting. This may indicate that part of the regulation is not working as expected. In addition, comparison of parameters between groups revealed that parameters $k_{I}, k_{L}, \beta, \tau_{d}, \tau_{a c h}$, and $M_{P}$ changed significantly between groups. These results were obtained using ANOVA analysis using 20 data sets for each group. Data from 10 subjects ( 2 experiments per subject) were analyzed. It should be noted that these results were obtained without any applying any model and parameter reduction techniques. Seen from a physiological point of view observing differences in the given parameters are reasonable, reduction in $k_{I}$ and $k_{L}$ indicate that with age and hypertension, the firing rate sensitivity to changes in pressure is reduced, increase of $\beta$ and $\tau_{d}$, indicate that with age and hypertension, the delay in onset of sympathetic response is increased and that parasympathetic dampening of the sympathetic response is attenuated, finally $\tau_{a c h}$ increase with age, but decrease with hypertension. An age related increase in $\tau_{a c h}$ suggests that with age it takes longer for the parasympathetic response to reach its maximum effect, while the decrease with hypertension, may be compensating for the fact that the vessels are significantly stiffer. Finally $M_{P}$ is increased with both age and hypertension indicating that parasympathetic regulation plays a more important role than subsequent sympathetic stimulation of heart rate.

Results from [22] (see Fig. 11 ) showed that there were significant differences between results from the STS and HUT procedures. Comparing the two tests showed a much larger increase in heart rate during HUT than during STS and a more significant drop in blood pressure during STS than during HUT, leading to more pronounced changes in firing rate and sympathetic/parasympathetic tone. Another noticeable difference is the change in the area of the hysteresis loop: the loop is significantly wider during STS than during HUT. Finally, we noticed that during HUT heart rate decrease during the initial preparation to tilt (before any blood pressure drop was observed), while during STS heart rate increased before the subject changed posture (the latter observation was also found in our first study [21]. This initial drop in heart rate is associated with a slight increase in blood pressure (compare panels A and E). This may be due to a short increase in venous return due to hydrostatic pressure difference imposed between the heart and the head. On the other hand the increase in heart rate immediately before standing may, as explained earlier, be due to vestibular and muscle sympathetic activation.

In addition to analysis of the results, in [21] we used sensitivity analysis (see Figs. 7 and 8) to rank model parameters from the most to the least sensitive. Re- 

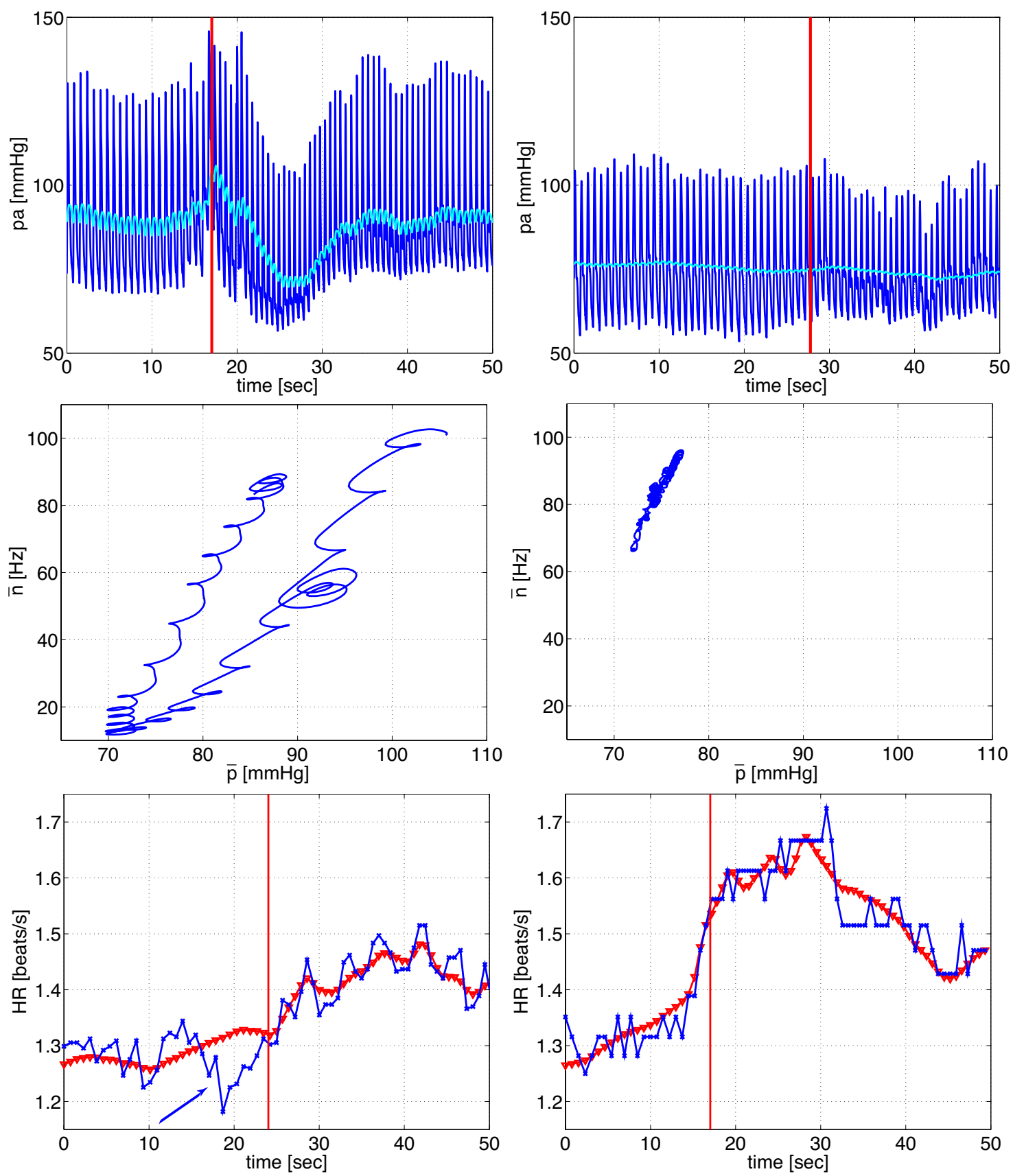

Figure 11: Blood pressure $[\mathrm{mmHg}$, firing rate $[\mathrm{Hz}]$, and heart rate $[\mathrm{bps}]$ versus time [sec] for STS (left panel) and for HUT experiment (right panel). Red curves are model results and blue curves are data. 
sults of this analysis showed that some parameters are very insensitive including $\tau_{I}, k_{I}, \zeta_{S}$, and $\tau_{a c h}$. Results varying a sensitive parameter, an intermediate parameter and an insensitive parameter are showed together with the ranking. One aspect not done for this study is to analyze if any of the sensitive parameters are correlated, more analysis is needed to investigate potential correlations. Such correlations are likely to exist, some initial attempts to study those have been done by Fowler [7] comparing results obtained with Nelder-Mead with both implicit filtering and using a genetic algorithm to optimize model parameters.

\subsection{Closed Loop model}

Closed loop cardiovascular models were investigated in [19, 20, 6, 4, 31]. All of these manuscripts discussed a model similar to the one shown in Fig. 5. In [19] and [20] we analyzed a model including all but two compartments (this model did not separate arteries and veins in the upper and lower torso). Instead the model included a compartment representing arteries in the hand. This model used heart rate data as an input to predict blood flow velocity and cerebral blood pressure during STS. In this study all model parameters were optimized and the model was validated against data from one healthy young subject. In [6] we used sensitivity analysis to show that cerebral blood flow velocity and arterial blood pressure data could be predicted using a simpler model not including the left atrium and the finger compartment, and when analyzing data during steady state (i.e., only modeling dynamics during during sitting), we showed that arteries and veins in the upper and lower body could be lumped together. Again, this model only used data from one healthy young subject. Furthermore, it should be noted that none of these model included a description of the diaphragm movement. Diaphragm movement was included in [4] (see Fig. 12), where we used airflow data to predict the movement. This study was carried out using a cardiovascular-respiratory model, that included both the systemic and pulmonary circulations. Note, this model was not designed to predict changes during STS, thus again fewer compartments were included separating the systemic circulation between upper and lower body. The latter model was developed to study dynamics during a $\mathrm{CO}_{2}$ challenge for a subject with congestive heart failure. Finally in [31] we developed a methodology for identifying a small subset of parameters that can be estimated given data during sitting. To do so, we used an even more simple model including only five compartments: the left ventricle, systemic arteries and veins, and cerebral arteries and veins. Again this model used heart rate as an input to predict dynamics of cerebral blood flow velocity and arterial blood pressure. Since this 

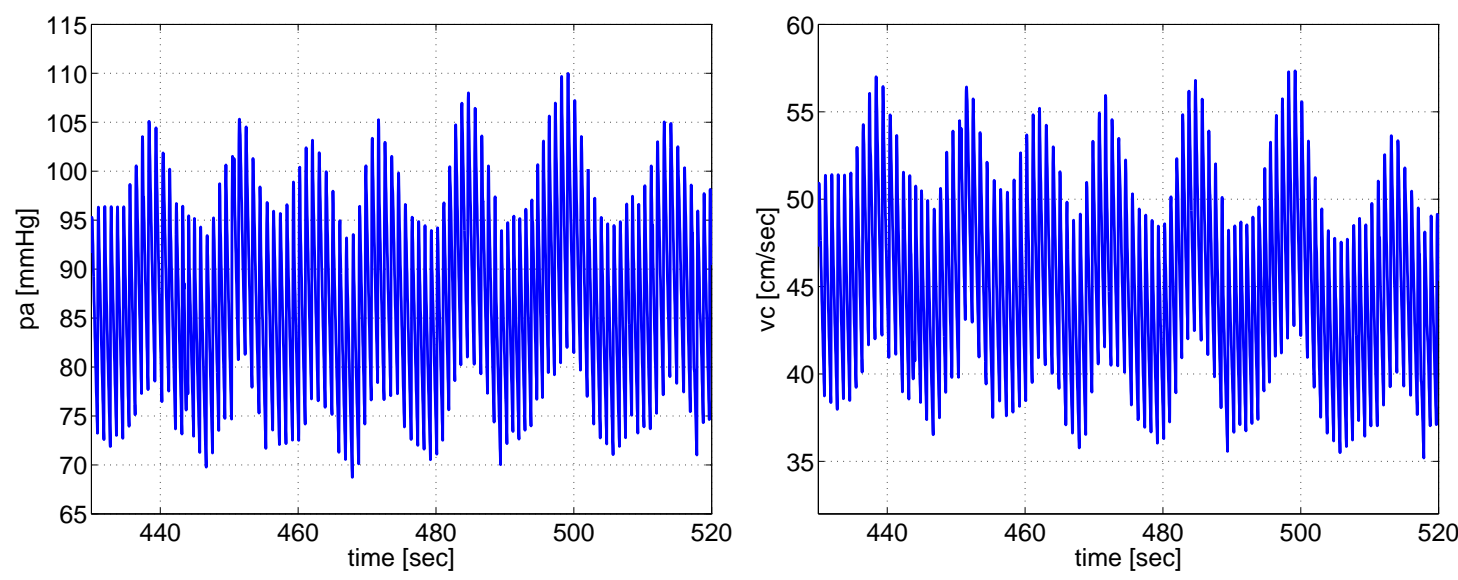

Figure 12: Model generated arterial pressure (top panel) and cerebral blood velocity (lower panel) when respiratory effects are included. The resulting curves are realistic and in accordance with measurements

model was significantly simpler, and because we used subset selection, we were able to predict dynamics for two groups of subjects including 16 healthy young subjects and 16 healthy elderly subjects. Results of this comparison showed that both cerebral resistance and compliance were modulated by aging. Results also showed that the total resistance was increased and that $T_{M}, f$ time for systolic pressure was increased.

All closed loop models were able to predict both blood pressure and cerebral blood flow velocity as shown in Fig. 13, however, only models were subset selection and sensitivity analysis were used could be analyzed against several datasets. The more advanced models (including more compartments) simply had to many parameters, thus making it very tedious to achieve good predictions of the data. In future work, we plan to apply subset selection to the more complex models, and with this method we anticipate that even the more complex models can be validated against multiple datasets, allowing identification and prediction of biomarkers.

The results shown in Fig. 13 were obtained with the complex control model developed in $[19,20]$. In this figure we show how the closed loop compartmental model allows prediction of arterial blood pressure $\left(p_{a}\right)$ and cerebral blood flow velocity $\left(v_{c}\right)$ during postural change from sitting to standing. The figures shows the overall estimation of these quantities and three "zooms" showing the prediction during sitting (for $t \in[45: 50] \mathrm{sec}$ ), during the transition (for $t \in[65: 70] \mathrm{sec}$ ), 

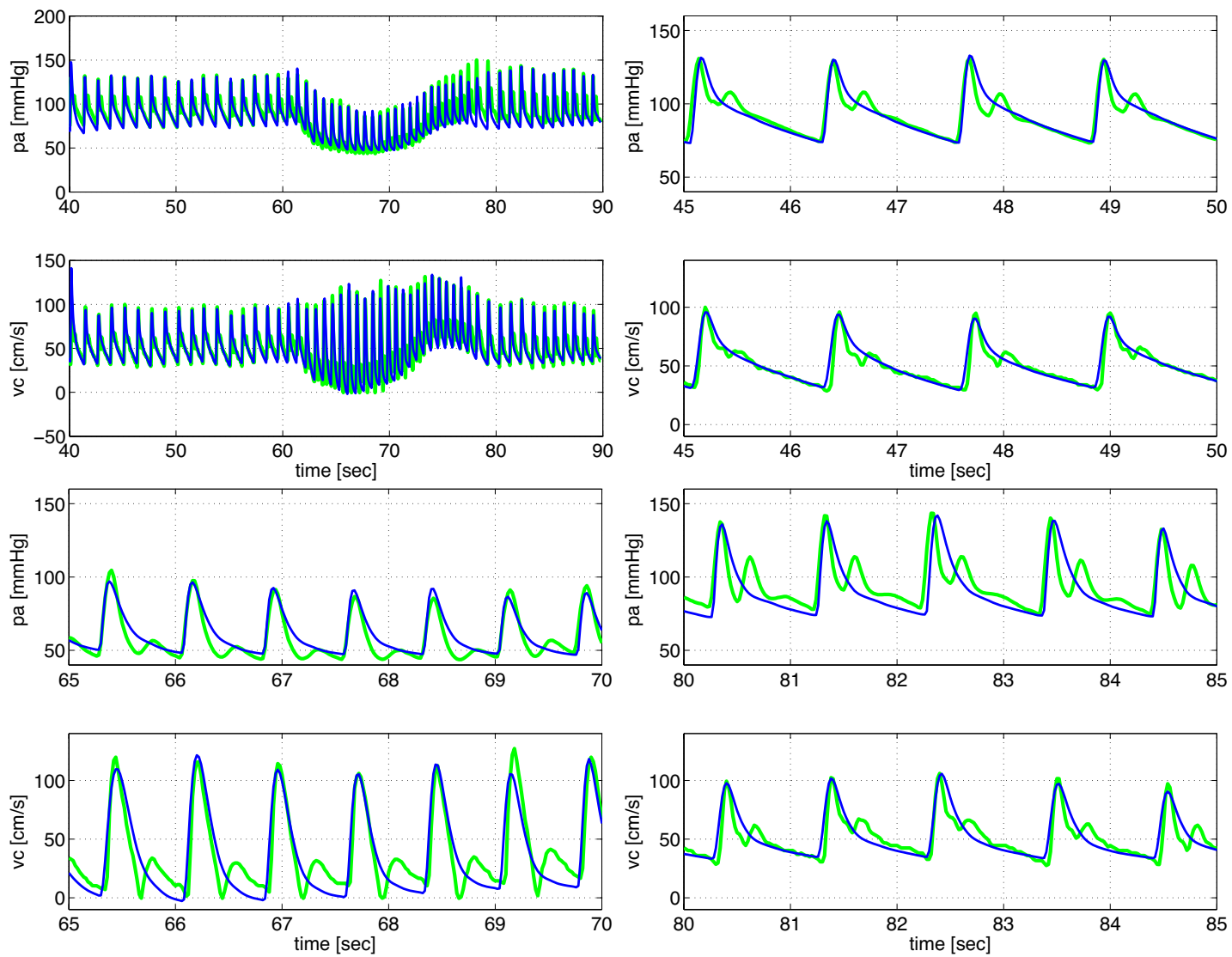

Figure 13: Pressure [mmHg] (top panel) and flow velocity [cm/sec] (bottom pannel) for STS with respiratory mechanical effect included.

and during standing (for $t \in[80: 85] \mathrm{sec}$ ). Note, while the model was able to predict the amplitude and approximate shape of the waveform, this type of model cannot predict the wave reflections observed in the data. To predict these, it is necessary to use a fluid dynamics model, that more realistically allows modeling of the wave propagation.

Another important aim with these models is to analyze internal dynamics of the states not measured. Results of this analysis is shown in Fig. 14. The first figure shows prediction of arterial blood pressure and cerebral blood flow velocity modeling the movement of the diaphragm as a function of measured airflow velocity. This result was obtained using a more elaborate closed loop compartmental model accounting for both vascular movement of blood and respiratory dynamics 
of $\mathrm{CO}_{2}$ and $\mathrm{O}_{2}$, this model is described in [5, 4]. The second figure (Fig. 14) shows not only effect on arterial pressure but on the internal states as well. It should be noted that respiration also affects the overall flow distribution and favors some braches at the expense of others and moreover Starlings law of the heart follows as a result. Furthermore, including mechanical coupling with respiration also affect model parameter values. Especially, parameters related to the ventricles and the control mechanisms are sensitive to effect of respiration. Surprisingly the impact of mechanical movement giving rise to respiration is somehow strong as see in Fig. 14, where the movement of the diaphragm during respiration is seen to be significant.

\subsection{Syncope}

Control mechanisms of the cardiovascular system play an important role in adaptation to postural changes during everyday activities. Syncope (meaning a pause in music) is the medical term for temporary loss of consciousness or fainting. Syncope is a common and significant medical problem that accounts for 3-6\% of emergency rooms visits and hospital admissions every year and in some cases may hallmark a significant underlying morbidity. Syncope is multifactorial and can be triggered from many different inputs into the central and peripheral autonomic systems. It often manifest as an abrupt loss of muscle tone with/without falling accompanied by a decline in blood pressure, blood flow to the brain, and heart rate, the latter may even lead to temporary cardiac arrest. This presentation may have clinical prodromes and changes in autonomic nervous system firing for minutes before the actual loss of consciousness occurs. Syncope is considered to be a reflex mechanism, protecting the vital organs (mainly the brain) from lack of perfusion.

It should be noted that the term syncope is related to a broad range of problems, and mechanisms related to triggering of syncope are mulifcatorial and different mechanisms can present in the same patients on various occasion not well understood. The most common type of syncope is neurally mediated syncope, which is characterized by peripheral vasodilation and a decrease in blood pressure (hypotension) along with a slowing of heart rate (bradycardia) or increasing heart rate (tachycardia). The result is temporary insufficient blood flow to the brain. The event is usually initiated by a withdrawal of peripheral sympathetic tone in upright posture, releases of vasoconstriction or active vasodilatation, accompanied by a decline in blood pressure. Cardioacceleration and central vasodilatation are compensatory mechanisms that may fail if blood pressure declines further. 

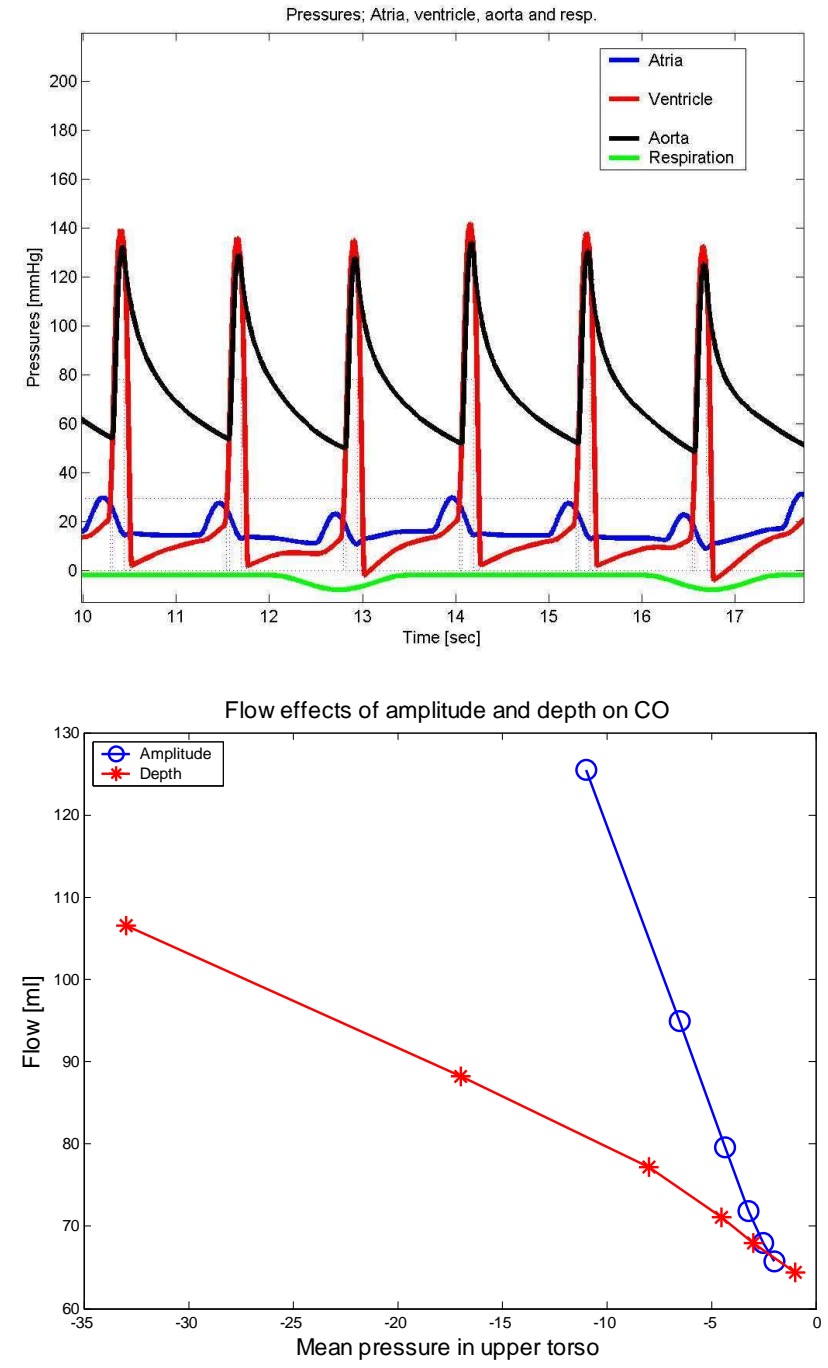

Figure 14: Upper panel shows atria, ventricular, aortic, and respiratory pressure $[\mathrm{mmHg}]$ versus time [sec] when respiration is included. Lower panel shows average flow through the heart (cardiac output) [ml] as a function of mean pressure, results show impact of changing respiration amplitude and depth (mean), respectively. 
Bradycardia or slow heart rate typically occurs later when blood pressure falls below certain threshold. In the model of HUT syncope appear approximately after 30 minutes as a sudden incident as a result of a crash in the control system where the effect of the controls saturates.

In the example discussed here the reduction in flow is initiated by the sudden drop in blood pressure observed during HUT, followed by compromised autonomic and cerebral autoregulation. In contrast to the heart rate regulation a unifying description of pressure changes shows that multiple simultaneous control mechanisms may be important in order to understand the experiments.

There are many reasons to use the model to analyze questions related to syncope, most importantly, it is not well know what mechanisms triggers syncope. A number of theories put forward to explain the phenomena [15]. How each of these theories impact pressure, flow, and HR dynamics could be studied using the proposed models.

To model dynamics during syncope we used the model illustrated in Fig. 5 modified to include intermediate control mechanisms essential for describing dynamics of HUT experiments over longer time scales ( $>35 \mathrm{~min}$ ). To do so it is important to account for dynamics of the venous pump, functioning to prevent pooling of flow in the extremities due to effects of gravity. This type of dynamics was not included in the original closed loop model, which was developed to study dynamics over a short time scale $(<2 \mathrm{~min})$. To model this we included a fluid shift compartment at the level of the legs. Through this compartment fluid was continuously transfered from the cardiovascular system to the extravascular environment. Consequently, during regulation, a combination of control mechanisms including arterial resistance and venous compliance regulation, fluid shift and dead volumes are regulated in this study. In addition, we incorporated a slight tension of the diaphragm The resulting model nicely describes the HUT experiments as shown in Fig. 15. In particular notice, how well the model reproduce data. The crash happens in the model when the arterial compliance regulation reach its saturation. Thus the heart rate cannot manage to regulate the system alone, venous return declines and the system breaks down resulting in syncope.

\section{Conclusion}

Above we have discussed a number of methodologies that can be used to develop patient specific models that allow prediction of arterial blood pressure, cerebral blood flow velocity, and heart rate during STS and HUT. Two model approaches 

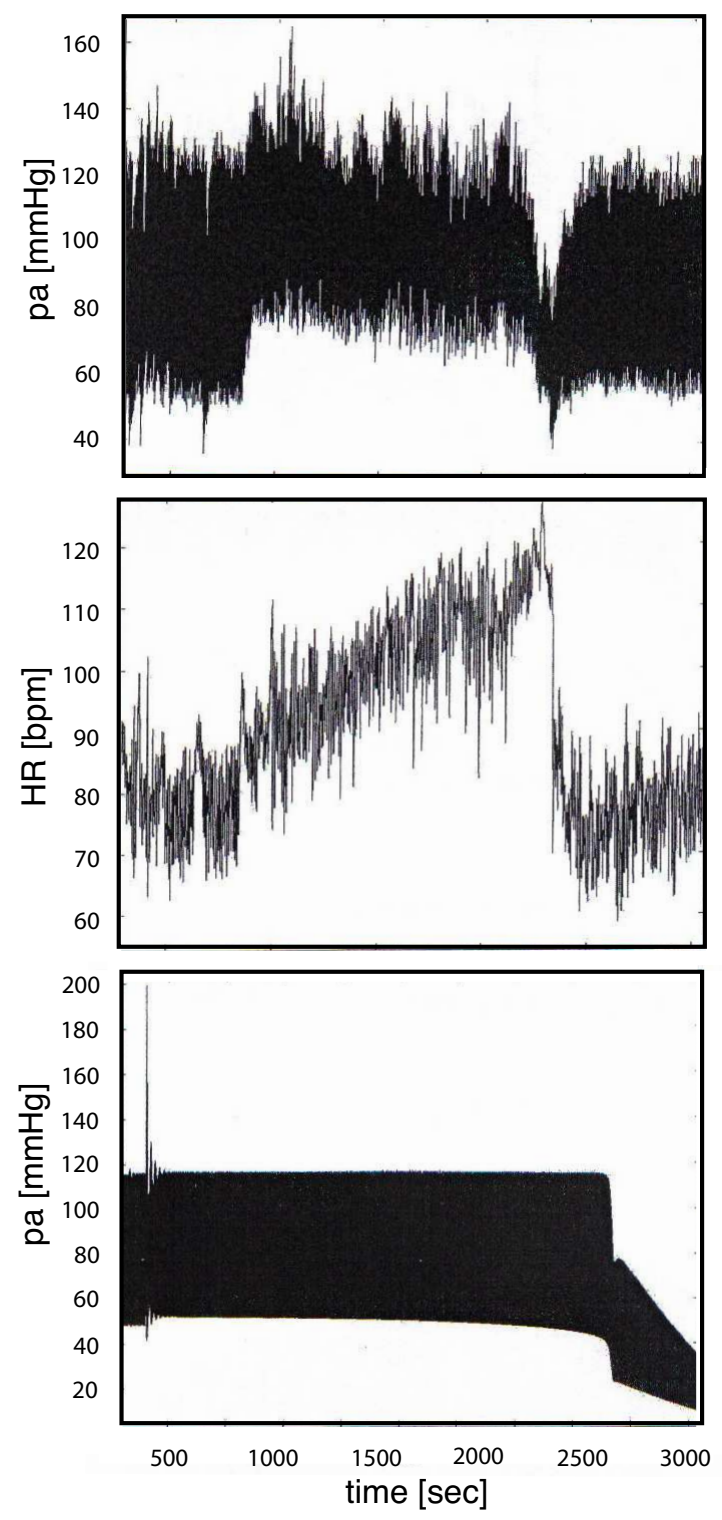

Figure 15: Upper panel shows arterial pulsatile pressure $[\mathrm{mmHg}]$ versus time [sec], center panel shows HR [bpm], and bottom panel shows results from a simulation. Results are shown during a HUT experiment. Note that the control mechanisms reach their saturation causing the syncope. 
were discussed open-loop models that use blood pressure as an input to predict heart rate, and a closed loop model that allow prediction of both blood pressure and blood flow velocity. We showed how these models could be used to predict patient specific data by solving the inverse problem estimating a set of model parameters that allow prediction of patient specific dynamics. Two analysis tecniques were investigated, and prove to be essential to obtain good results: sensitivity analysis, which allow us to rank model parameters from the most to the least sensitive, and subset selection, which allow us to identify correlation among model parameters. The sensitivity ranking can be used to identify insensitive parameters that cannot be estimated given data, and allowed us to identify essential components of the model. Subset selection allowed us to predict a subset of uncorrelated parameters among the sensitive parameters, which can be reliably be estimated using the available data. Finally, we show how these models can be used to model clinical events (syncope), and using this example, we have shown that this type of modeling approach has potential to be used for better understanding of underlying mechanisms triggering syncope. Another important outcome is that the type of models developed here allow prediction of internal states, which cannot be measured experimentally.

However, the models themselves have a number of limitations. First, the sparsity of data used for prediction of dynamics in the closed loop models discussed above, is probably the main limitation is obtaining good patient specific models. As discussed earlier, only 4-6 parameters can be estimated reliably given these data. Another limitation, not of the models, but of the patient specific analysis is that without measurements of cardiac output it is difficult to get flow distributions correct. Many different estimates of cardiac output can be distributed to obtain the correct arterial pressure and cerebral blood flow velocity, and as discussed in [31], the models analyzed here typically underestimates cardiac output. Consequently, one important suggestion we can put forward for researchers interested in using the proposed modeling tools discussed here is to incorporate some measurements of cardiac output.

Another important lesson we learned (discussed in [6]), is that for any given application, it is essential to include as few compartments as necessary to model the proposed dynamics, and then be conservative in reducing the number of parameters estimated to predict patient specific dynamics.

Finally, if one is interested in studying dynamics of the wave form, the ODE compartmental models should be coupled with a fluid dynamics model allowing accurate prediction of the reflected waves. 


\section{Acknowledgements}

The authors wish to thank graduate students involved with this project including Laura Ellwein, Dept of Biomedical Engineering, Marquette University, Scott Pope, SAS Corp, Raleigh, NC, April Alston, Dept of Math, NCSU, and numerous undergraduate students participating in research and REU program at NCSU. Furhtermore, authors would like to thank Hien Tran and Tim Kelley, Department of mathematics, NCSU. The work by M.S. Olufsen was supported in part by NSFDMS grant \# 0616597 and NSF-OISE grant\# 0437037. V. Novak, director of the SAFE Laboratory at BIMDC was supported by NIH-NIA Harvard Older American Independence Center 2P60 AG08812-11A1, Core B.

\section{References}

[1] Allen L.J.S. An Introduction to Mathematical Biology. Pearson Education, Up Sadddle River, NJ, 2003.

[2] Danielsen M. Modeling offeedback mechanisms which control the heart function in a view to an implementation in cardiovascular models. $\mathrm{PhD}$ Thesis, Roskilde University, Denmark. Text No 358, 1998.

[3] DeVault K., Gremaud P., Zhao P., Vernieres G., Novak V., and Olufsen M.S. Blood flow in the circle of Willis: Modeling and calibration. SIAM J Multiscale Anal Simul, 7:888-909, 2008.

[4] Ellwein L.M. Cardiovascular and Respiratory Regulation, Modeling and Parameter Estimation.. PhD Thesis, Applied Mathematics, NC State University, Raleigh, NC. 2009.

[5] Ellwein L.M., Pope S.R., Xie A., Batzel J.J., Kelley C.T., and Olufsen M.S. Modeling cardiovascular and respiratory dynamics in congestive heart failure. Submitted, 2009.

[6] Ellwein L.M., Tran H.T., Zapata C., Novak V., and Olufsen M.S. Sensitivity analysis and model assessment: Mathematical models for arterial blood flow and blood pressure. J Cardiovasc Eng, 8:94-108, 2008.

[7] Fowler K.R., Gray G.A., and Olufsen M.S. Modeling heart rate regulation Part II: Parameter identification. J Cardiovasc Eng, 8:109-119, 2008. 
[8] Guyton A.C. and Hall J.E. Textbook of medical physiology. WB Saunders, Philadelphia, PE, 9th ed, 1996.

[9] Johnson P., Shore A., Potter J.F., Panerai R.B., and James M. Baroreflex sensitivity measured by spectral and sequence analysis in cerebrovascular disease - methodological considerations Clin Auton Res, 16: 270-275, 2006.

[10] Jose A. and Collison D. The normal range and determinants of the intrinsic heart rate in man. Cardiovasc Res, 4: 160-167, 1970.

[11] Kaufmann H., Biaggioni I., Voustianiouk A., Diedrich A., Costa F., Clarke R., Gizzi M., Raphan T., and Cohen B. Vestibular control of sympathetic activity: An otolith-sympathetic relfex in humans. Exp Brain Res, 143: 463-469, 2002.

[12] Kyrylov V., Severyanova L.A., and Vieira A. Modeling Robust Oscillatory Behaviour of the Hypothalamic-Pituitary-Adrenal Axis. IEEE Trans Biomed Eng, 52:1977-1983, 2005.

[13] Liang K.H., Krus D.J., and Webb J.M. K-fold crossvalidation in canonical analysis. Multivariate Behavioral Resarch, 30:539-546, 1995.

[14] Low P.A. and Bannister R.G. Multiple System Atrophy and Pure Autonomic Failure. In: Low P.A.(eds). Clinical Autonomic Disorders. Lippincott-Raven Publishers, Philadelphia, PE, pp. 555-575, 1997.

[15] Mosqueda-Garcia R., Furlan R., Tank J., and Fernandez-Violante R. The elusive pathophysiology of neurally mediated syncope. Circulation, 102: 28982906, 2000.

[16] Olufsen M.S. Structured tree outflow condition for blood flow in larger systemic arteries. Am J Physiol, 276:H257-H268, 1999.

[17] Olufsen M.S., Nadim A., and Lipsitz L.A. Dynamics of cerebral blood flow regulation explained using a lumped parameter model. Am J Physiol, 282:R611-R622, 2002.

[18] Olufsen M.S. and Nadim A. On deriving lumped models for blood flow and pressure in the systemic arteries. Math Biosci Eng, 1:61-80, 2004. 
[19] Olufsen M.S., Tran H.T., and Ottesen J.T. Modeling cerebral blood flow control during posture change from sitting to standing. Cardiovasc Eng, 4(1):47$58,2004$.

[20] Olufsen M.S., Ottesen J.T., Tran H.T., Ellwein L.M., Lipsitz L.A., and Novak V. Blood pressure and blood flow variation during postural change from sitting to standing: model development and validation. J Appl Physiol, 99:15231537, 2005.

[21] Olufsen M.S., Tran H.T., Ottesen J.T., Lipsitz L.A., and Novak V. Modeling baroreflex regulation of heart rate during orthostatic stress. Am J Physiol, 291:R1355-R1368, 2006.

[22] Olufsen M.S., Alston A.V., Tran H.T., Ottesen J.T., and Novak V. Modeling heart rate regulation, Part I: Sit-to-stand versus head-up tilt. J Cardiovasc Eng, 8:73-87, 2008.

[23] Opthof T. The normal range and determinants of the intrinsic heart rate in man. Cardiovasc Res, 45:173-176, 2000.

[24] Ottesen J.T. Modeling of the baroreflex-feedback mechanism with timedelay. J Math Biol, 36:41-63, 1997.

[25] Ottesen J.T. Nonlinearity of baroreceptor nerves. Surv Math Ind, 7:187-201, 1997.

[26] Ottesen J.T. General Compartmental Models of the Cardiovascular System in Mathematical Modelling in Medicine. IOS press, Amsterdam, pp. 121-138, 2000 .

[27] Ottesen J.T. Modeling the dynamical baroreflex-feedback control. Math Comp Mod, 31:167-173, 2000.

[28] Ottesen J.T. and Danielsen M. Modeling ventricular contraction with heart rate changes. $J$ Theor Biol, 222:337-346, 2003.

[29] Ottesen J.T. Valveless pumping in a fluid-filled closed elastic tube-system: one-dimensional theory with experimental validation. J Math Bioll, 46:309$332,2003$.

[30] Ottesen J.T., Olufsen M.S., and Larsen J. Applied mathematical models in human physiology. SIAM, Philadelphia, PA, 2004. 
[31] Pope S., Ellwein L.M., Zapata C.L., Novak V., Kelley C.T., and Olufsen M.S. Estimation and identification of parameters in a lumped cerebrovascular model. Math Biosci Engl, 6:93-115, 2009.

[32] Pope S.R. Parameter identification in lumped compartment Cardiorespiratory models. PhD Thesis, Applied Mathematics, NC State University, Raleigh, NC. 2009.

[33] Ray C. Interaction of the vestibular system and baroreflexes on sympathetic nerve activity in humans. Am J Physiol, 279:H2399-H2404, 2000.

[34] Ray C. and Monahan K. The vestibulosympathetic reflex in humans: Neural interactions between cardiovascular reflexes. Clin and Exp Pharmaco and Physiol, 29: 98-102, 2002.

[35] Ray C. and Carter J. Vestibular activation of sympathetic nerve activity. Acta Physiol Scand, 177: 313-319, 2003.

[36] Robbe H.W.J., Mulder L.J.M., Ruddel H., Langewitz W.A., Weldman J.B.P., Mulder G. Assessment of baroreceptor reflex sensitivity by means of spectral analysis. Hypertension, 10: 538-543, 1987.

[37] Robertson D., Low P.A., and Polinsky R.J. Primer on the autonomic nervous system. Academic Press, Boston, MA, 2nd ed, 2005.

[38] Smith J.J. and Kampine J.T. Circulatory physiology, the essentials. Williams and Wilkins, Baltimore, MD, 3rd ed, 1990.

[39] Steele B., Olufsen M.S., and Taylor C. Fractal network model for simulating abdominal and lower extremity blood flow during rest and exercise conditions. Comp Meth Biomech Biomed Eng, 10:39-51, 2007.

[40] Strogatz S.H. Nonlinear Dynamics and Chaos. Perseus Books Publishing, LLC, 1994.

[41] Ursino M. and Lodi C.A. A simple mathematical model of the interaction between intracranial pressure and cerebral hemodynamics. J Appl Physiol, 82:1256-1269, 1997.

[42] Ursino M. and Magosso E. Short-term autonomic control of cardiovascular function: a mini-review with the help of mathematical models. $J$ Integr Neurosci, 2:219-247, 2003. 
[43] Ursino M. and Magosso E. Short-term autonomic control of the cardiorespiratory system: a summary with the help of a comprehensive mathematical model. IEEE Eng MEd Biol Soc, 1:354-358, 2006.

[44] Wilson T.D., Cotter L.A., Draper J.A., Misra S.P., Rice C.D., Cass S.P., and Yates B.J. Vestibular inputs elicit patterned changes in limb blood flow in conscious cats. J Physiol, 575: 671-684, 2006.

[45] Zhang R., Zuckerman J.H., Iwasaki K., Wilson T.E., Crandall C.G., and Levine B.D. Autonomic Neural Control of Dynamic Cerebral Autoregulation in Humans. Circulation, 106:1814-1820, 2002.

[46] Zhang R. and Levine B.D. Autonomic Ganglionic Blockade Does Not Prevent Reduction in Cerebral Blood Flow Velocity During Orthostasis in $\mathrm{Hu}-$ mans. Stroke, 38:1238-1244, 2007. 